\title{
A theoretical framework for microscopic surface and interface dipoles, work functions and valence band alignments in 2D and 3D halide perovskite heterostructures
}

\section{Supporting Information}

Boubacar Traoré, ${ }^{11}$ Pooja Basera, ${ }^{1,2}$ Alexandra J. Ramadan, ${ }^{3}$ Henry J. Snaith, ${ }^{3}$ Claudine Katan, ${ }^{1}$ and Jacky Even*2

1 Univ Rennes, ENSCR, CNRS, ISCR - UMR 6226, F-35000 Rennes, France.

2 Univ Rennes, INSA Rennes, CNRS, Institut FOTON - UMR 6082, F-35000 Rennes, France.

${ }^{3}$ Clarendon Laboratory, Department of Physics, University of Oxford, Oxford, United Kingdom

E-mail: boubacar.traore@univ-rennes1.fr

jacky.even@insa-rennes.fr

\section{Contents}

Text S1 | Computational details for localized basis-sets calculations (SIESTA) . . . . . . . . . . . 2

Text S2 | The computation of absolute valence energy levels $\ldots \ldots \ldots \ldots$

Text S3 | Definition of model parameters . . . . . . . . . . . . . . . . . . . . . . . . 5

Text S4 $\mid \mathrm{CsPbl}_{3}$ and $\mathrm{MAPbl}_{3}$ relaxed structures $\ldots \ldots \ldots \ldots \ldots \ldots$

Text S5 | Absolute valence energy alignment of $\mathrm{CsPbBr}_{3}$ perovskite $\ldots \ldots \ldots \ldots$. . . . . . . . 8

Text S6 | Interpretation of the effect of surface relaxation on the absolute valence energy level $\ldots \ldots 10$

Text S7| Relaxed structures with surface coating using $\mathrm{Cs} \ldots \ldots \ldots \ldots$

Text S8 | Surface passivation with DMSO . . . . . . . . . . . . . . . . . . . . . . . . 14

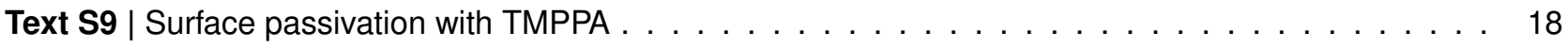

Text S10 | Additivity of the surface dipoles in $(\mathrm{PEA})_{2} \mathrm{Pbl}_{4} / \mathrm{CsPbl}_{3}$ heterostructure $\ldots \ldots \ldots \ldots$ 


\section{Text S1 | Computational details for localized basis-sets calculations (SIESTA)}

DFT-based calculations ${ }^{1,2}$ were performed using SIESTA ${ }^{3,4}$ using a basis set of finite-range of numerical atomic orbitals. For structural optimization, we used the van der Waals density functional with $\mathrm{C} 09$ exchange ${ }^{5}$ within the van der Waals DF2 flavor to describe the exchange-correlation term (we modified SIESTA version "masterpost-4.1-251" to include DF2 flavor in which $Z_{a b}=-1.887$ instead of $Z_{a b}=-0.8491$ in DF1). This tuning was suggested to improve C09 based geometry optimization over that with the original DF1 flavor ${ }^{6}$. The latter has already proven to provide a good description of experimental lattice constants similar to those obtained with optimized GGA based PBEsol functional in solids. ${ }^{7,8}$. Norm-conserving Troullier-Martins pseudopotentials were used for each atomic species to account for the core electrons. ${ }^{9} 1 s^{1}, 2 s^{2} 2 p^{2}, 2 s^{2} 2 p^{3}, 5 s^{2} 5 p^{5}, 5 d^{10} 6 s^{2} 6 p^{2}, 2 s^{2} 2 p^{4}$ and $3 s^{2} 3 p^{4}$ were used as valence electrons for $\mathrm{H}, \mathrm{C}, \mathrm{N}, \mathrm{I}, \mathrm{Pb}, \mathrm{O}$ and $\mathrm{S}$ respectively. Polarized Double- $\zeta$ (DZP) basis set with an energy shift of $200 \mathrm{meV}$ and a real space mesh grid energy cutoff of 300 Rydberg were used for the calculations. We used $8 \times 8 \times 8$ and $6 \times 6 \times 1$ Monkhorst-Pack k-point grids for the bulk and slab systems, respectively. For $\mathrm{CsPbl}_{3}$ slabs, we doubled the cells along a-b planes resulting in lattice constants equal to that of the bulk multiplied by $\sqrt{2}\left(a_{s / a b}=\sqrt{2} \times a_{b u l k}\right)$.

For band structures, band alignments and charge densities for polarization density profiles, we used singlepoint calculations with GGA-PBE ${ }^{10}$ functional combined with DFT-1/2 ${ }^{11}$ including spin-orbit coupling on top of vdwDF2-C09 optimized structures. Spin-orbit coupling (SOC), when used, was considered in its on-site approximation as proposed by Fernández-Seivane et al ${ }^{12}$.

To compute the polarization density profiles, we removed the spurious "unphysical" charge densities beyond 30 Bohr from the surface deep inside the vacuum. Since, so far, SIESTA does not keep symmetries from geometry optimizations, we also take the half-sum of the total charge densities across the slab in forward and reverse directions resulting in a more symmetrized density profile (e.g. : $\rho[1: n]=0.5 \times(\rho[1: 1: n]+\rho[n:-1: 1])$ where $n$ is the size the charge density array $\rho$ along $z$ ). This to reflect the symmetry of the surfaces by construction and has only minor effects on the results.

\section{Structural relaxation}

The fully relaxed bulk structures were used to construct the slabs. With the slabs constructed, only the atomic positions were allowed to relax when surface layers relaxation was requested. We only allowed the two surface octahedral layers of the perovskite to relax while keeping the bulk-like regions frozen to the positions of the optimized bulk structure. This strategy allows capture of the surface effects during the relaxation while maintaining the integrity of the bulk region of the slabs, which is useful for the energy level alignment. We used the fast inertial relaxation engine (FIRE) algorithm ${ }^{13}$ for the different relaxations and the maximum force was set to $0.04 \mathrm{eV} / \mathrm{A}$. We used 10 octahedral layers to construct the different slabs since we found that this thickness was sufficient for converged absolute valence energy levels. For the $\mathrm{Pbl}_{2}$ terminated surface, we had 9 effective octahedral layers 
due to the removal of surface Csl atoms to obtain $\mathrm{Pbl}_{2}$ termination. The same applies for $\mathrm{Cspbr}_{3}$ slabs (vide infra)

\section{Structural information}

For the structures, we used the pseudo-cubic structure of $\mathrm{CsPbl}_{3}$ generated from its room-temperature othorhombic cell as reported in Ref. ${ }^{14}$. The lattice constant of this pseudo-cubic structure was obtained using : $a_{\text {pseudocubic }}=\left(V_{\text {ortho }} / 4\right)^{1 / 3}$, where $V_{\text {ortho }}$ is the volume of the room-temperature orthorhombic cell. Similarly, for $\mathrm{CsPbBr}_{3}$, we used the pseudo-cubic structure from its room-temperature orthorhombic cell ${ }^{15}$. For the slab models, we constructed ten octahedral layer systems from the relaxed pseudo-cubic structures. We found that 10 octahedral layers were sufficient for obtaining converged bulk-like quantities.

\section{D/3D heterostructure construction}

We used the doubled pseudo-cubic cells in the plane of the perovskite to lattice match it to the 2D layer cell. This cell doubling corresponds to multiplying the pseudo-cubic lattice constant by $\sqrt{2}$. In order to mimic the structural properties of 3D bulk materials at room temperature, we used Cs as a pseudotom instead of $\mathrm{FA}\left(\mathrm{FAPbl}_{3}{ }^{16}\right.$, $\mathrm{FA}=$ formamidinium). Hence, we replaced $\mathrm{FA}$ with $\mathrm{Cs}$ which we referred to as $\mathrm{CsPbl}_{3}$ under the heterostructure section of the main manuscript. For the heterostructure using 2D based $B A$, we used the room temperature structure of $\mathrm{BA}_{2} \mathrm{Pbl}_{4}$ as reported in Ref. ${ }^{17}$. As for PEA, we used the structure recorded at $295 \mathrm{~K}$ from Ref. ${ }^{18}$. We considered 9 octahedral layers of $\mathrm{CsPbl}_{3}$ to mimic the 3D part of the heterostructure. The in-plane lattice constants of the 3D part were imposed for the whole heterostructure and were not allowed to relax during the 2D/3D interface geometry optimization. We only allowed the c-lattice constant along $z$ to relax along with the atomic positions.

\section{Note on slab dipole correction}

In modeling slabs with asymmetric surface terminations, an artificial electric field is created across the slab due to the periodic boundary conditions. This is reflected as a potential slope in the vacuum region of the slab. Quite often a dipole correction scheme is imposed to compensate for the artificial electric field ${ }^{19,20}$ and thus making the potential flat in the vacuum region. This would then allow to compute quantities such as work functions with asymmetric surface terminations. Here, we used symmetrically terminated slab surfaces by construction such that there is negligible potential slope in the vacuum region and that there is no need to use any dipole correction scheme. We checked that the dipole correction has negligible effects on the absolute valence energy levels with our symmetric surface terminations. In addition the symmetric termination is desired in our approach in order to reliably extract the surface dipoles from the developed methodology. 


\section{Band gap correction}

Since normal DFT severely underestimates the bandgap, we used the revisited Slater half-occupation technique in the so-called DFT-1/2 implementation in order to correct the band gaps ${ }^{11,21}$. This technique allows to obtain reasonable band gaps in good agreement with experiments and at a much computationally affordable cost. For DFT- $1 / 2$ correction, we removed $1 / 2 e^{- \text {from }} \mathrm{p}$ and $\mathrm{s}$ orbitals of halides and lead $(\mathrm{Pb})$ atoms, respectively. This is because the valence band maximum of these halide perovskites is made of an anti-bonding hybridization between $\mathrm{Pb}(\mathrm{s})$ and halides $(\mathrm{p})$ states $^{22}$. We note that spin-orbit coupling effect was included.

\section{Charge transfer}

The charge transfer from the adsorbate to the slab or vice-versa was computed using:

$$
\Delta \rho=\rho_{\text {slab+adsorb }}-\left(\rho_{\text {slab }}+\rho_{\text {adsorb }}\right)
$$

where $\Delta \rho$ is the charger transfer from or to the perosvkite slab, $\rho_{s / a b+a d s o r b}$ is charge density of the whole system including the perovskite and the adsorbate layers, $\rho_{s / a b}$ is the charge density of the perovskite part of the slab and $\rho_{\text {adsorb }}$ is the charge density of the adsorbate or added foreign layer. Here, a positive value of $\Delta \rho$ corresponds to electronic charge accumulation while a negative value corresponds to electronic charge depletion. Note that within SIESTA convention for total charge density: a positive sign is for electronic charge density and a negative sign is for ionic charge density.

\section{Text S2 | The computation of absolute valence energy levels}

The absolute valence energy levels of the different systems were computed using the Hartree potential alignment with the vacuum level set as the reference ${ }^{23,24}$. Here, the absolute valence energy level $E_{v}^{a b s}$ is obtained using:

$$
E_{v}^{a b s}=V B M_{\text {bulk }}+\Delta V_{H}+\Delta E_{\text {vacuum }}
$$

where $V B M_{\text {bulk }}$ is the valence band maximum of the bulk system, $\Delta V_{H}=V_{H}^{\text {slab }}-V_{H}^{\text {bulk }}$ is the shift of the Hartree potential between the slab and the bulk systems, and $\Delta E_{\text {vacuum }}=0-E_{\text {vacumm }}$ is the vacuum level shift with respect to zero energy level. For the conduction band, we added to $E_{v}^{a b s}$ the theoretically computed band gap of the bulk compound using DFT-1/2. This then gives,

$$
E_{c}^{a b s}=E_{v}^{a b s}+E_{g, b u l k}
$$


a

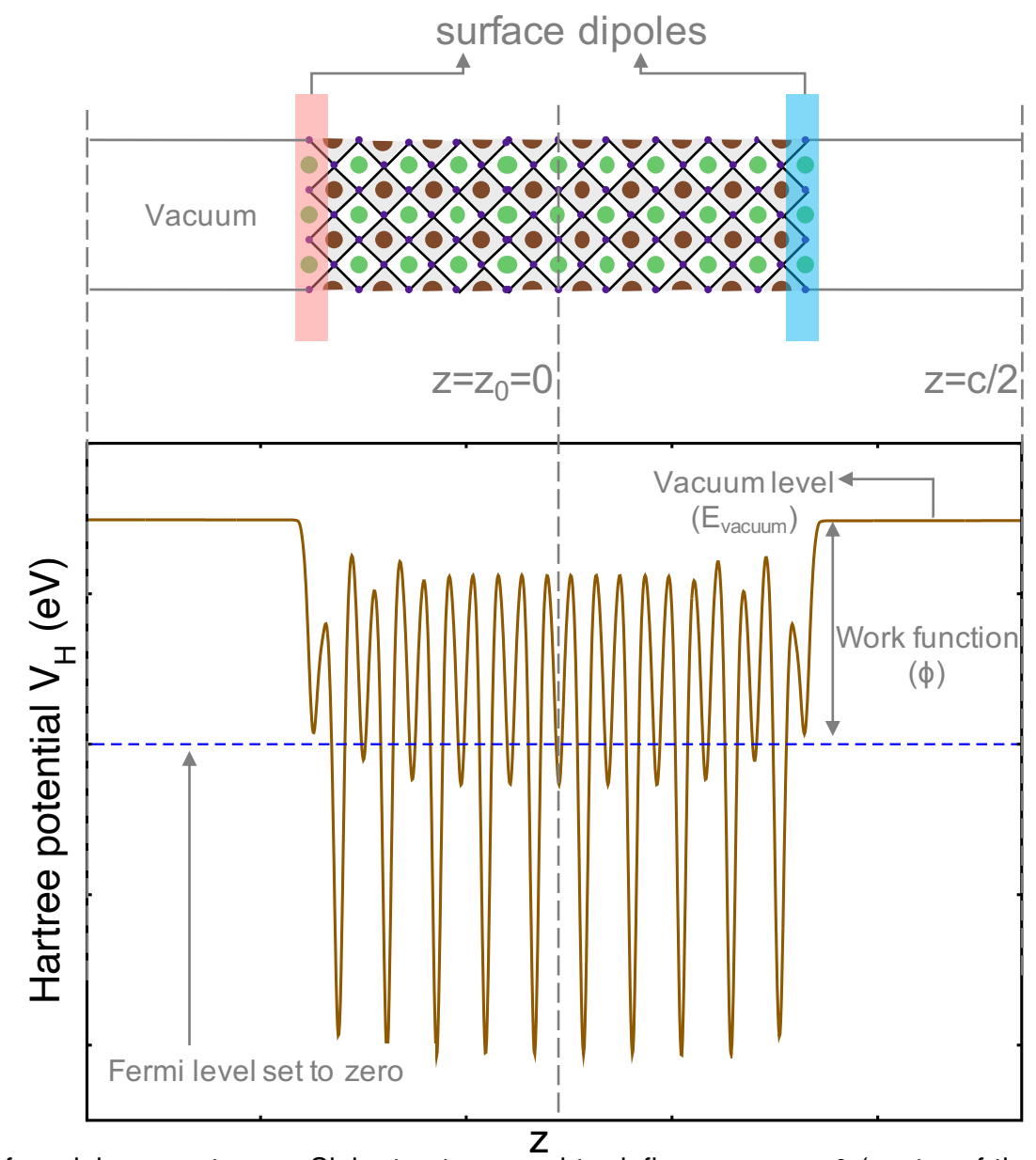

Figure S1 | Definition of model parameters. a Slab structure used to define $z=z_{0}=0$ (center of the slab) and $z=c / 2$ (middle of the vacuum) for the derivation of the surface dipoles. By construction, we use symmetrical terminations on either side of the surfaces such that the dipole moment is vanishing in the middle bulk like region at $z=z_{0}$. $\mathbf{b}$ Definition of the work function from a Hartree potential profile. 
$\mathrm{CsPbl}_{3}$

a

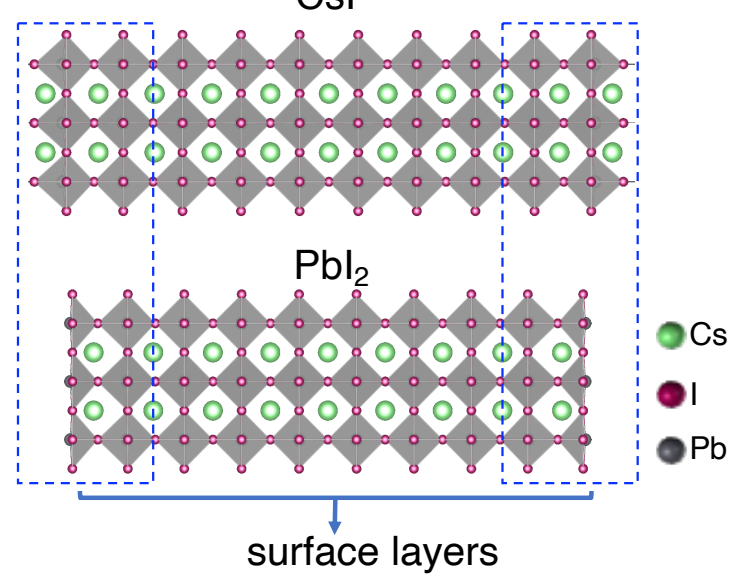

b

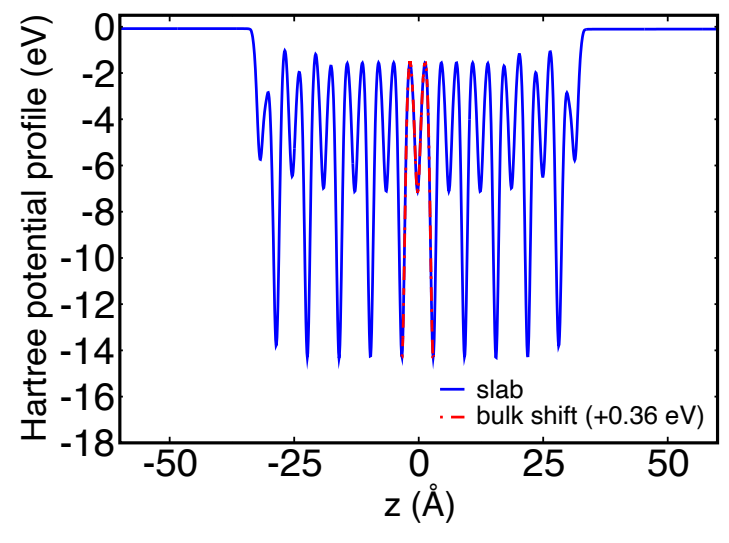

Figure S2 | a CsPbl 3 structures with $\mathrm{Csl}$ and $\mathrm{Pbl}_{2}$ terminations with relaxed surface layers. The surface layers are shown by dashed rectangles on either side of the slab. $\mathbf{b}$ Hartree potential alignment method used to obtain the absolute valence energy levels of the systems. The profile of the slab corresponds to the relaxed Csl terminated surface shown in $\mathbf{a}$. For this example, a potential shift $\left(\Delta V_{H}\right)$ of $+0.36 \mathrm{eV}$ between the slab and the bulk results in superimposed profiles at the center of the slab (bulk-like region).

\section{$\mathrm{MAPbl}_{3}$}

MAI : unrelaxed surface

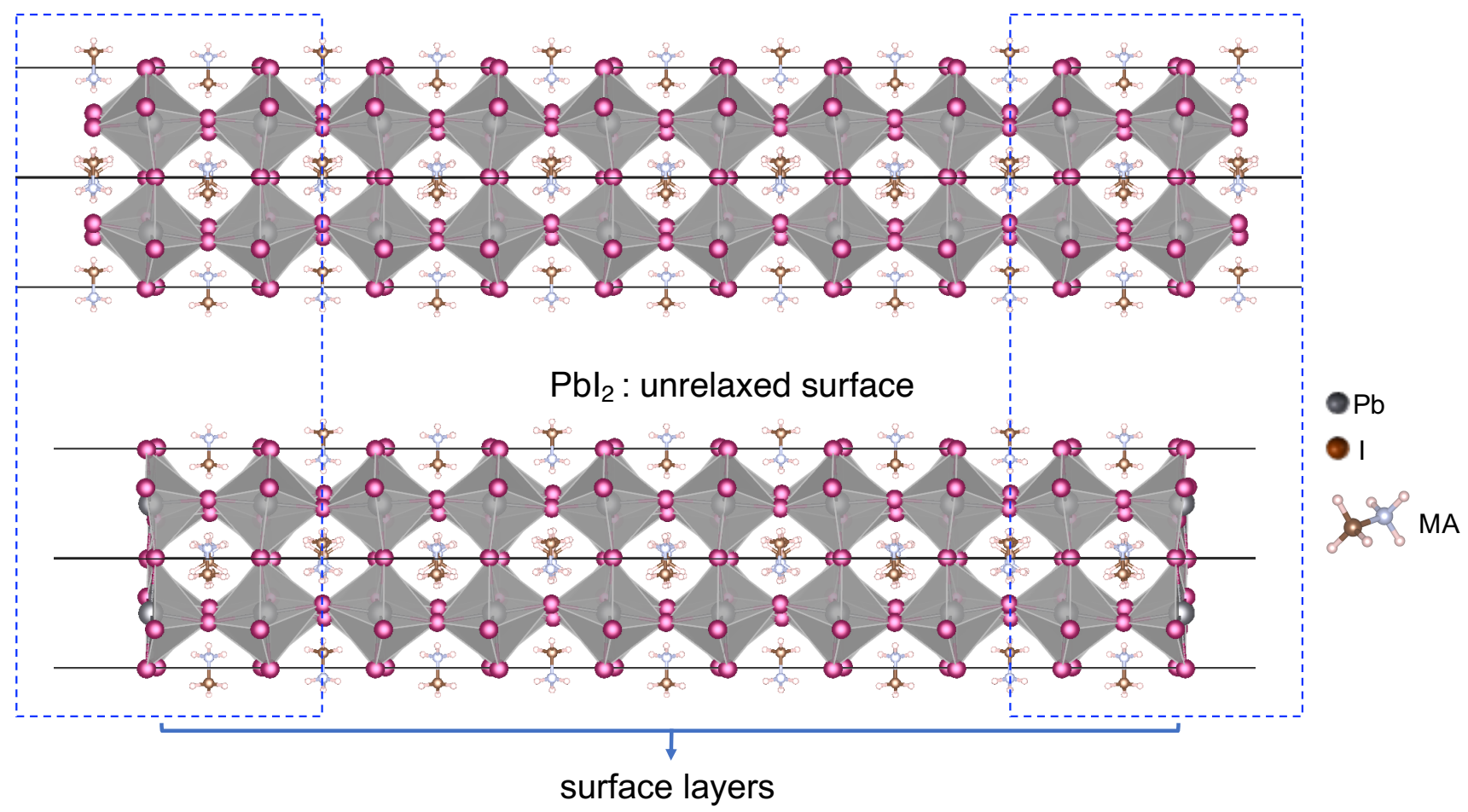

Figure S3 | Structures of $\mathrm{MAPbl}_{3}$ slabs with the relaxed surface layers considering both $\mathrm{MAl}$ and $\mathrm{Pbl}_{2}$ terminations. 
MAI : relaxed surface

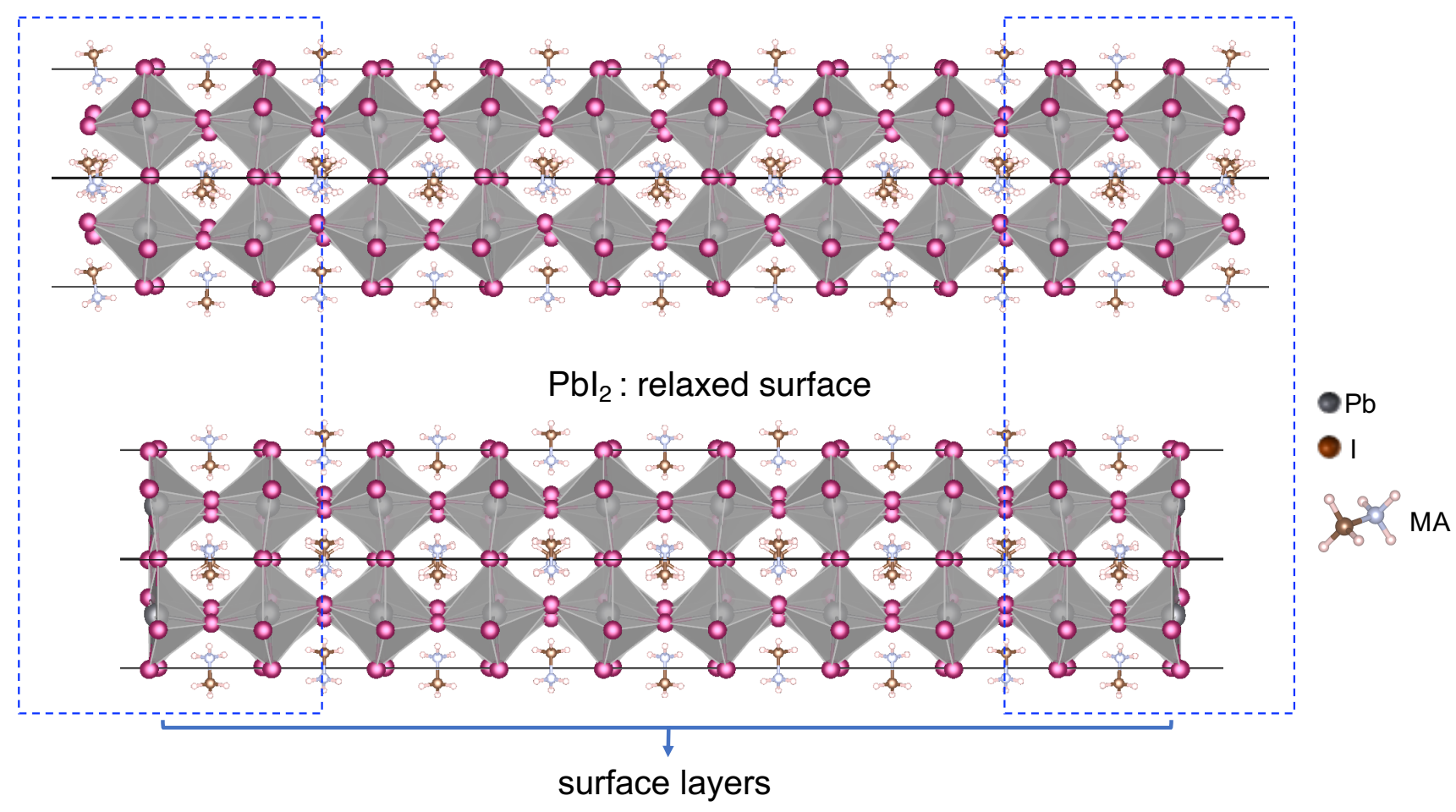

Figure $\mathbf{S 4} \mid$ Structures of $\mathrm{MAPbl}_{3}$ slabs with the relaxed surface layers considering both $\mathrm{MAl}$ and $\mathrm{Pbl}_{2}$ terminations. 


\section{Discussion on the relaxation effect on computed absolute valence energy levels}

At the surface of a crystalline solid, the termination of the crystal structure and the absence of the subsequent ions/atoms, implies that the lowest energy relative position of the surface atoms will be different as compared to the atoms within the infinite crystalline slab. We can allow the surface to "relax" computationally, and then estimate this impact upon the surface dipole for the different surface terminations. Hence, we compute the surface dipoles and $E_{v}^{a b s}$ of the different terminations for the relaxed surface layers of both slabs (Figure 1c,d of the main manuscript for $\mathrm{CsPbl}_{3}$ and Figure 1g,h for $\mathrm{MAPbl}_{3}$ ) and the results are summarized in Table 1 of the manuscript. Again, we find an excellent agreement between $\Delta \mathrm{E}_{v}^{a b s}$ and $\Delta \phi$ obtained from $\Delta p$. In the case of $\mathrm{MAPbl}_{3}$, surface relaxation slightly pushes $E_{v}^{a b s}$ down for both $\mathrm{MAl}$ and $\mathrm{Pbl}_{2}$ terminations with a minor effect on the sign and value of $\Delta \mathrm{E}_{v}^{a b s}$. However, contrary to the unrelaxed case, $E_{v}^{a b s}$ for Csl-termination is larger than that of $\mathrm{Pbl}_{2}$-termination in $\mathrm{CsPbl}_{3}$ slab. We obtain similar results for $\mathrm{CsPbBr}_{3}$ slabs as discussed in Text $\mathrm{S} 5$ (vide infra). This trend between the relaxed and unrelaxed surface layers can be anticipated from the lower surface dipole of $\mathrm{Pbl}_{2}$ as a result of surface relaxation. The changes are due to the combined effect of the octahedral distortions of the surface layer along with the contraction or the stretching of Pbl bond lengths at the surface (see Text S5). This shows that in these ionic semiconductors, surface relaxation impacts the surface dipoles, which in turn directly tunes the subsequent absolute energy alignments and the work functions. It also highlights the importance of experimental techniques giving access to the local atomistic details at the surfaces and interfaces to probe the relaxation of the perovskite lattice. Finally, we shall point out that the differences between the workfunctions obtained here for the relaxed and unrelaxed bare surfaces, may appear at first sight quite large. But in most practical cases, such surfaces are, in addition, likely to be covered intentionally or naturally by passivation layers as discussed in the main manuscript.

\section{Text S5 | Absolute valence energy alignment of $\mathrm{CsPbBr}_{3}$ perovskite}

This section compares the absolute valence energy alignment of $\mathrm{CsPbBr}_{3}$ slabs with the surface layers unrelaxed (Figure S5) or relaxed (Figure S6). Similar to $\mathrm{CsPbl}_{3}$, the trend of $E_{v}^{a b s}$ between $\mathrm{CsBr}$ and $\mathrm{PbBr}_{2}$ terminated surfaces is reversed when the surface layers are relaxed as compared to the unrelaxed ones. In the unrelaxed case, $E_{v}^{a b s}$ is deeper for the $\mathrm{PbBr}_{2}$ terminated surface as compared to $\mathrm{CsBr}$ by about $2.2 \mathrm{eV}$ and this is due to the larger surface dipole for the $\mathrm{PbBr}_{2}$ terminated surface (Figure S5c). Notably, the difference of $2.2 \mathrm{eV}$ in $E_{v}^{a b s}$ is in excellent agreement with $\Delta \phi(-2.20 \mathrm{eV})$ computed from the difference of the surface dipole moments. For the relaxed surface layers, $E_{v}^{a b s}$ is deeper for the $\mathrm{CsBr}$ terminated surface as compared to $\mathrm{PbBr}_{2}$ by about $1.03 \mathrm{eV}$. Again, this difference in nicely captured by the larger surface dipole of the CsBr-terminated surface as a result of surface relaxation (Figure S6c). By computing $\Delta \phi(+1.08 \mathrm{eV})$ using Eq. (10) of the main text, we observe an excellent agreement with $\Delta E_{v}^{\text {abs }}(+1.03 \mathrm{eV})$. Table $\mathbf{S} 1$ summarizes the computed absolute valence energy levels along with the difference of the surface dipoles for the various cases of $\mathrm{CspBr}_{3}$ slabs. 
Table S1 | Comparison of absolute valence energy levels $\left(E_{v}^{a b s}\right)$ calculated for the different systems. The table also compares the shifts of absolute valence energy levels obtained from the difference of surface dipoles $(\Delta \phi$ using Eq 10 of the main text) and those computed from the Hatree potential alignment (Figure 2b and Eq. 14 of the main text). CsBr-termination is taken as the reference.

\begin{tabular}{|c|c|c|c|c|}
\hline \multicolumn{5}{|c|}{ Unrelaxed surface layers } \\
\hline & $E_{v}^{a b s}=\phi(\mathrm{eV})$ & $\Delta \mathrm{E}_{V}^{a b s}(\mathrm{eV})$ & $\Delta p(\mathrm{C} / \mathrm{m})$ & $\Delta \phi(\mathrm{eV})$ \\
\hline CsBr & -4.80 & - & - & - \\
\hline $\mathrm{PbBr}_{2}$ & -7.00 & -2.20 & $1.97 \times 10^{-11}$ & -2.22 \\
\hline \multicolumn{5}{|c|}{ Relaxed surface layers } \\
\hline $\mathrm{CsBr}$ & -6.79 & - & - & - \\
\hline $\mathrm{PbBr}_{2}$ & -5.76 & +1.03 & $-0.96 \times 10^{-11}$ & +1.08 \\
\hline
\end{tabular}
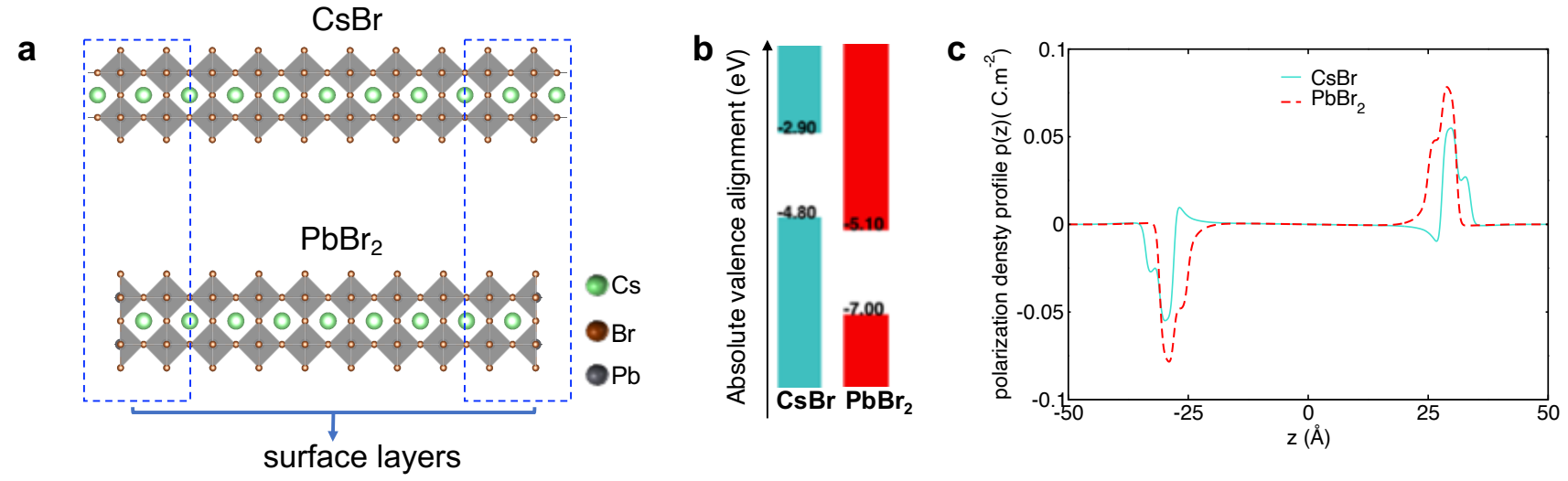

Figure S5 | Effect of surface termination on absolute energy alignments of $\mathrm{CsPbBr}_{3}$ with unrelaxed surface layers. a $\mathrm{CsPbBr}_{3}$ structures with $\mathrm{CsBr}$ and $\mathrm{PbBr}_{2}$ terminations with unrelaxed surface layers. The surface layers are shown by dashed rectangles on either side of the slab. b Computed absolute valence energy levels for $\mathrm{CsBr}$ and $\mathrm{PbBr}_{2}$ terminated surfaces, respectively for the unrelaxed surface layers. c Polarization density profiles of the two slab systems showing different surface dipoles for the unrelaxed surface layers. The integration of these profiles result in the surface dipole moments.

a

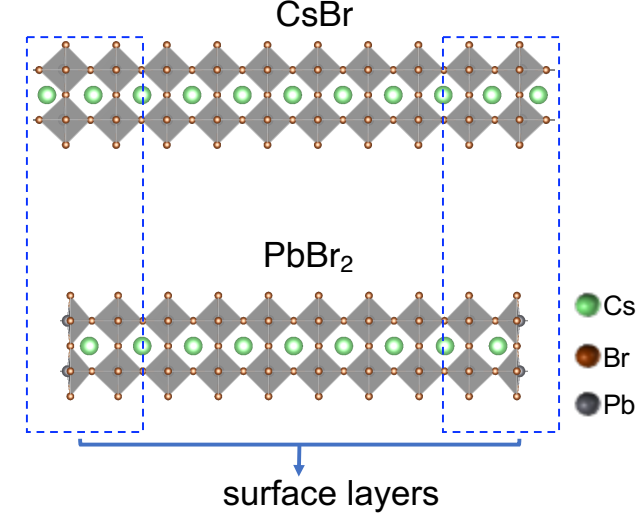

b

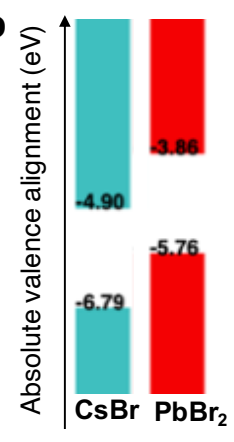

C

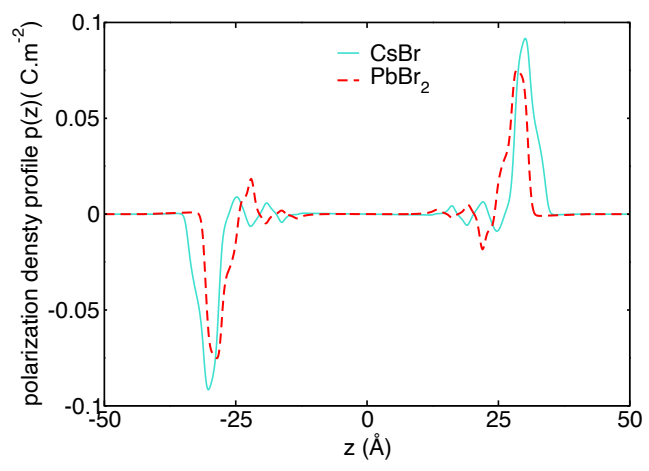

Figure S6 | Effect of surface termination on absolute energy alignments of $\mathrm{Cs} \mathrm{PbBr}_{3}$ with relaxed surface layers. a CsPbBr 3 structures with $\mathrm{CsBr}$ and $\mathrm{PbBr}_{2}$ terminations with relaxed surface layers. The surface layers are shown by dashed rectangles on either side of the slab. b Computed absolute valence energy levels for $\mathrm{CsBr}$ and $\mathrm{PbBr}_{2}$ terminated surfaces, respectively for the relaxed surface layers. c Polarization density profiles of the two slab systems showing different surface dipoles for the relaxed surface layers. The integration of these profiles result in the surface dipole moments. 


\section{Text S6 | Interpretation of the effect of surface relaxation on the absolute valence energy level}

Table S2 compares the variation of the in-plane and out-of-plane $\mathrm{Pbl}$ bond lengths and $\mathrm{Pb}-\mathrm{I}-\mathrm{Pb}$ angles for the $\mathrm{CsPbl}_{3}$ slabs when the surface layers are unrelaxed and relaxed. The results are shown for both $\mathrm{Csl}$ and $\mathrm{PbI}_{2}-$ terminated surfaces. Upon the surface layers relaxation, both the in-plane and out-of-plane Pbl bond lengths are stretched for Csl-termination while only the in-plane $\mathrm{Pbl}$ bond is stretched for $\mathrm{Pbl}_{2}$ termination with the outof-plane one contracted. However, the main impact of the surface relaxation is seen on the in-plane $\mathrm{Pb}-\mathrm{I}-\mathrm{Pb}$ angles which undergo a dramatic decrease from the ideal $180^{\circ}$ for both $\mathrm{Csl}$ and $\mathrm{Pbl}_{2}$ terminations. This effect is much larger in Csl termination reaching about twice the variation seen in $\mathrm{Pbl}_{2}$ termination. It is quite establisehd that octahedral distortions have a significant influence on the electronic properties of halide perovskites ${ }^{25-28}$. The valence band maximum (VBM) of these compounds is made of an anti-bonding hybridization between the halide $\mathrm{X}(\mathrm{p})$ states and the metal $\mathrm{M}(\mathrm{s})$ states. The larger $\mathrm{Pb}-\mathrm{I}-\mathrm{Pb}$ deviation from the ideal $180^{\circ}$ in the case of CsI termination leads to much less destabilized $X(p)-M(s)$ coupling, which results in much deeper VBM. In addition, the longer $\mathrm{Pbl}$ bonds in Csl-termination also exacerbates this less destabilized $X(p)-M(s)$ coupling at the VBM

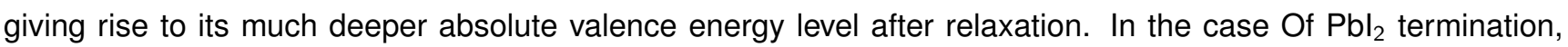
the same effect occurs but to a lesser extent. Besides, the shorter out-of-plane $\mathrm{Pbl}$ bond in $\mathrm{Pbl}_{2}$ after relaxation tends to destabilize $X(p)-M(s)$ coupling which counteracts the deepening of VBM. Thus, its less deeper absolute valence energy level after relaxation as compared to the Csl-termination. Therefore, the change of trend between the relaxed and unrelaxed surfaces for the two terminations is related to the way the two surfaces are reorganized after relaxation which is reflected in the computed surface dipoles.

Table S2 | Comparison of average in-plane and out-of-plane $\mathrm{Pb}-\mathrm{I}-\mathrm{Pb}$ angles, in-plane and out-of-plane Pbl bond lengths at the outermost surface layers of $\mathrm{CsPbl}_{3}$ slab. subscripts "in" and "out" refer to the in-plane and out-ofplane, respectively. The percentage variation with respect to the unrelaxed systems are given in parenthesis.

\begin{tabular}{|c|c|c|c|c|}
\hline & $\mathrm{Pbl}_{i n}(\AA)$ & $\mathrm{Pb}-\mathrm{I}-\mathrm{Pb}_{\text {in }}\left({ }^{\circ}\right)$ & $\mathrm{Pbl}_{\text {out }}(\AA)$ & $\mathrm{Pb}-\mathrm{I}-\mathrm{Pb}_{\text {out }}\left({ }^{\circ}\right)$ \\
\hline \multicolumn{5}{|c|}{ Unrelaxed surface layers } \\
\hline Csl & 3.15 & 180.0 & 3.15 & 180 \\
\hline $\mathrm{Pbl}_{2}$ & 3.15 & 180.0 & 3.15 & 180 \\
\hline
\end{tabular}

Relaxed surface layers

\begin{tabular}{lllll}
\hline \hline Csl & $3.17(+0.6 \%)$ & $165.7(-7.9 \%)$ & $3.20(+1.6 \%)$ & $180.0(0.0 \%)$ \\
$\mathrm{Pbl}_{2}$ & $3.16(+0.3 \%)$ & $172.6(-4.1 \%)$ & $3.11(-1.3 \%)$ & $180.0(0.0 \%)$ \\
\hline
\end{tabular}

Table S3 compares the variation of the in-plane and out-of-plane $\mathrm{Pbl}$ bond lengths and $\mathrm{Pb}-\mathrm{I}-\mathrm{Pb}$ angles for the $\mathrm{MAPbl}_{3}$ slabs when the surface layers are unrelaxed and relaxed. The results are shown for both $\mathrm{MAl}$ and $\mathrm{Pbl}_{2}-$ terminated surfaces. Here, the relaxation did not induce significant changes on the average Pbl bond lengths and $\mathrm{Pb}-\mathrm{I}-\mathrm{Pb}$ angles for either surface termination. However, the values undergo a noticeable fluctuation between the lower and upper bounds as shown in the table, as a result of the distortions that lead to some deepening of the subsequent absolute valence energy levels. However, no change of the trend is seen between the unrelaxed 
and relaxed surfaces for the two terminations due to the similar fluctuations and distortions induced for the two systems.

Table S3 | Comparison of the in-plane and out-of-plane Pb-I-Pb angles, in-plane and out-of-plane Pbl bond lengths at the outermost surface layers of $\mathrm{MAPbl}_{3}$ slab. subscripts "in" and "out" refer to the in-plane and out-ofplane, respectively. The range between the lower and upper bounds is rather shown here to show the distortion effects since the average values for the relaxed structures were similar to the unrelaxed ones.

\begin{tabular}{|c|c|c|c|c|}
\hline & $\mathrm{Pbl}_{i n}(\AA)$ & $\mathrm{Pb}-\mathrm{I}-\mathrm{Pb}_{i n}\left(^{\circ}\right)$ & $\mathrm{Pbl}_{\text {out }}(\AA)$ & $\mathrm{Pb}-\mathrm{I}-\mathrm{Pb}_{\text {out }}\left({ }^{\circ}\right)$ \\
\hline \multicolumn{5}{|c|}{ Unrelaxed surface layers } \\
\hline MAI & 3.18 & 146.3 & 3.23 & 160.3 \\
\hline $\mathrm{Pbl}_{2}$ & 3.18 & 146.3 & 3.23 & 160.3 \\
\hline \multicolumn{5}{|c|}{ Relaxed surface layers } \\
\hline MAI & $3.14-3.22$ & $140.5-152.5$ & $3.20-3.32$ & $160.2-160.5$ \\
\hline $\mathrm{Pbl}_{2}$ & $3.11-3.26$ & $142.2-150.0$ & $3.08-3.20$ & $160.3-160.6$ \\
\hline
\end{tabular}




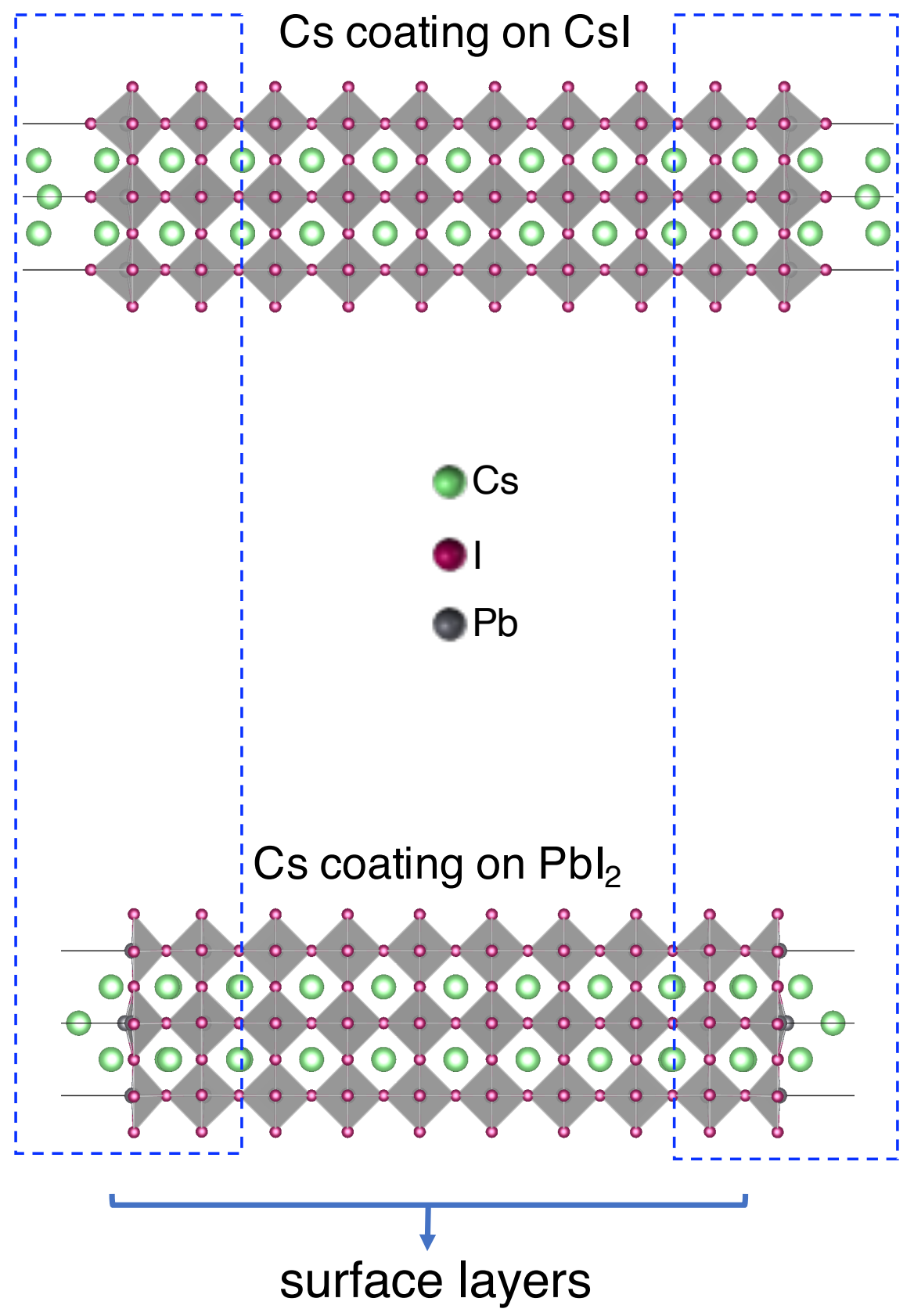

Figure S7 | Comparison of relaxed $\mathrm{CsPbl}_{3}$ structures with $\mathrm{Csl}$ and $\mathrm{Pbl}_{2}$ terminations with the surface layers coated with a monolayer of Cs. The surface layers are shown by dashed rectangles on either side of the slab.

Figures $\mathbf{S} 8$ and $\mathbf{S 9}$ report the band structures of $\mathrm{CsPb}_{3}$ slab with relaxed Csl-terminated surface and the slab terminated with a monolayer of Cs, respectively. As can be seen from Figure S9, the monolayer of Cs introduce shallow surface states at the conduction band with the Fermi level $\left(E_{F}\right)$ crossing the bands and making the system n-type. From the local density of states (LDOS) shown in Figure S9, the bands due to the surface states are indeed localized on Cs monolayers. 


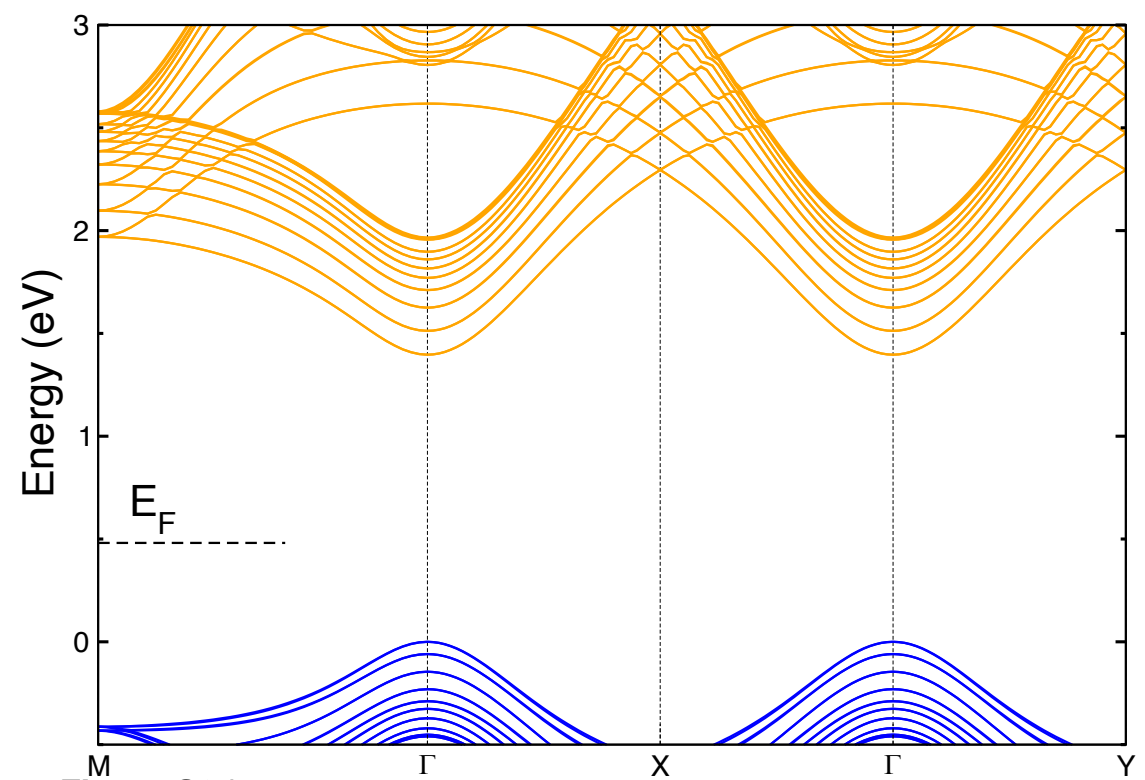

Figure S8 | Band structure of relaxed Csl-terminated $\mathrm{CsPb}_{3}$ slab surface.

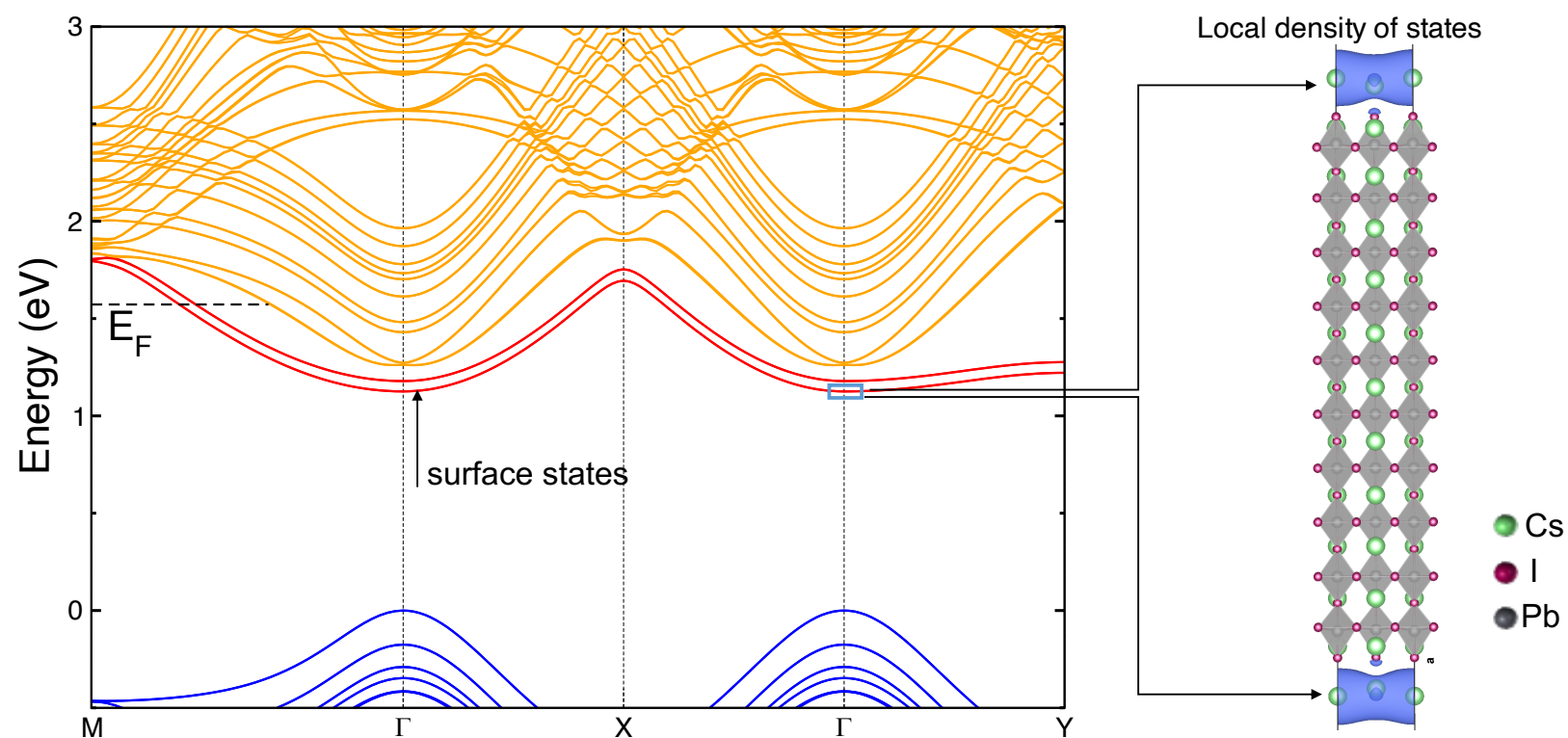

Figure S9 | (Left) Band structure of $\mathrm{CsPbl}_{3}$ slab with a monolayer of Cs placed at the surfaces. (Right) Local density of states (LDOS) of the surface states from the band structure. The LDOS is integrated over an energy window around $\Gamma \mathrm{k}$-point as shown by the small blue box. The isosurface level is $3.0 \times 10^{-6}$. 


\section{Text S8 | Surface passivation with DMSO}

To insert DMSO at the surface, we place the oxygen $(\mathrm{O})$ atom of the molecule at the position of an outer terminal I (iodine) atom ${ }^{29}$. In doing so, we remove a neighboring Cs atom or MA molecule to keep the charge neutrality of the system. In these slabs, the pristine surfaces contains 2 outer I atoms and we substitute one of them by DMSO. Hence, we have a ratio of one DMSO molecule per 2 outer I atoms. The relaxation of the surface with DMSO induces octahedral distortions of the surface Pbl layers (see Figure S7, S8 for the relaxed systems). For instance, the in-plane $\mathrm{Pb}-\mathrm{I}-\mathrm{Pb}$ angles of the relaxed $\mathrm{CsPbl}_{3}$ surface layers with $\mathrm{DMSO}$ on the Csl-termination vary between $\sim 141^{\circ}$ and $\sim 164^{\circ}$ from the ideal undistorted $180^{\circ}$. These octahedral distortions cause a widening of the band gap in the surface region as can be seen from the layer-by-layer resolved projected density of states (PDOS) of the system in Figure S9. Nevertheless, the resulting band structure exhibits dispersive bands with no surface states inside the gap showing the passivating role of DMSO (Figure S13, S14).

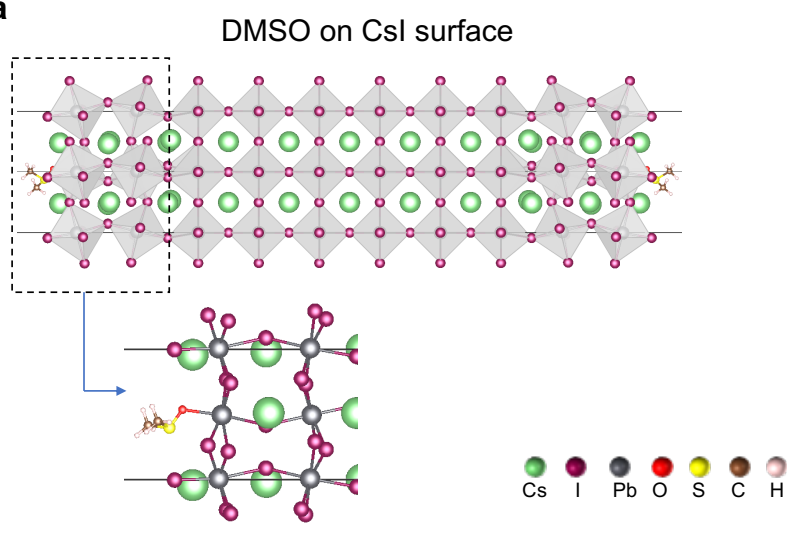

b

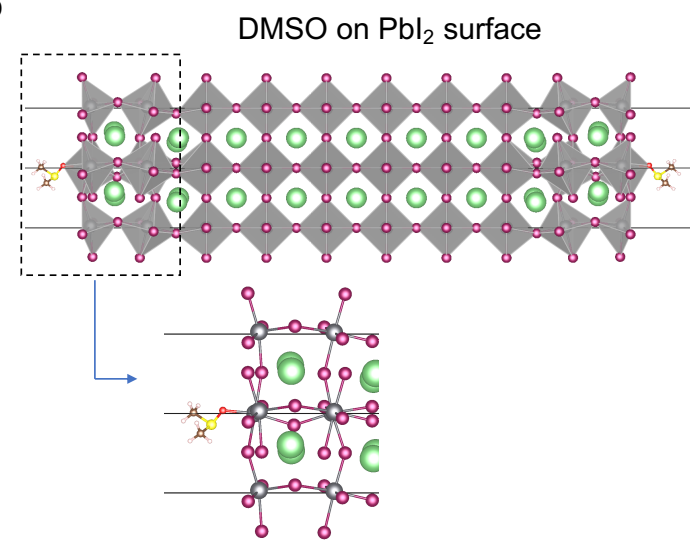

Figure S10 | Relaxed $\mathrm{CsPbl}_{3}$ slabs with DMSO on ' a the Csl-terminated surface $\mathbf{b}$ the $\mathrm{Pbl}_{2}$-terminated surface.

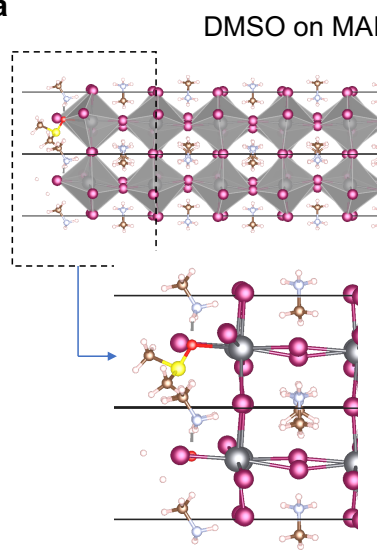

b DMSO on $\mathrm{Pbl}_{2}$ surface

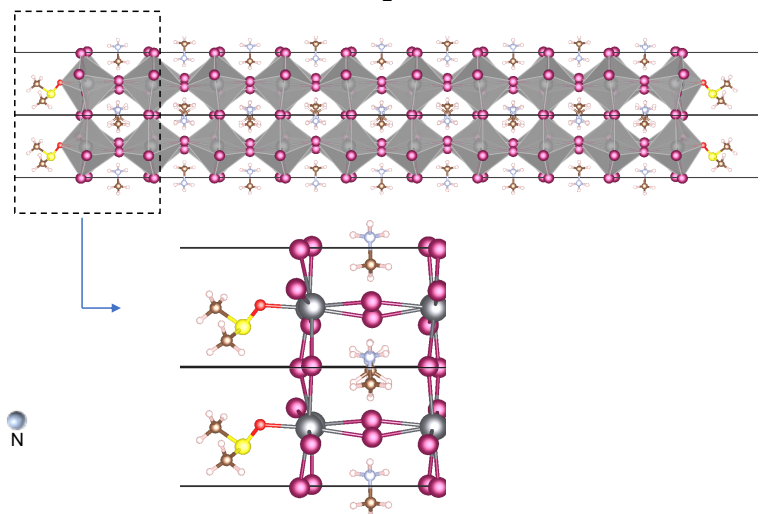

Figure S11 | Relaxed $\mathrm{MAPbl}_{3}$ slabs with DMSO on ' $\mathbf{a}$ the MAl-terminated surface $\mathbf{b}$ the $\mathrm{Pbl}_{2}$-terminated surface. 


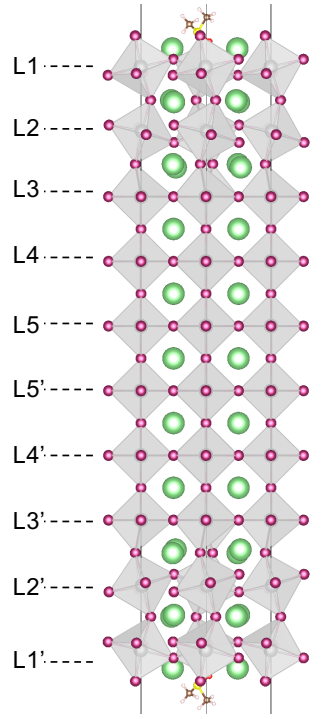

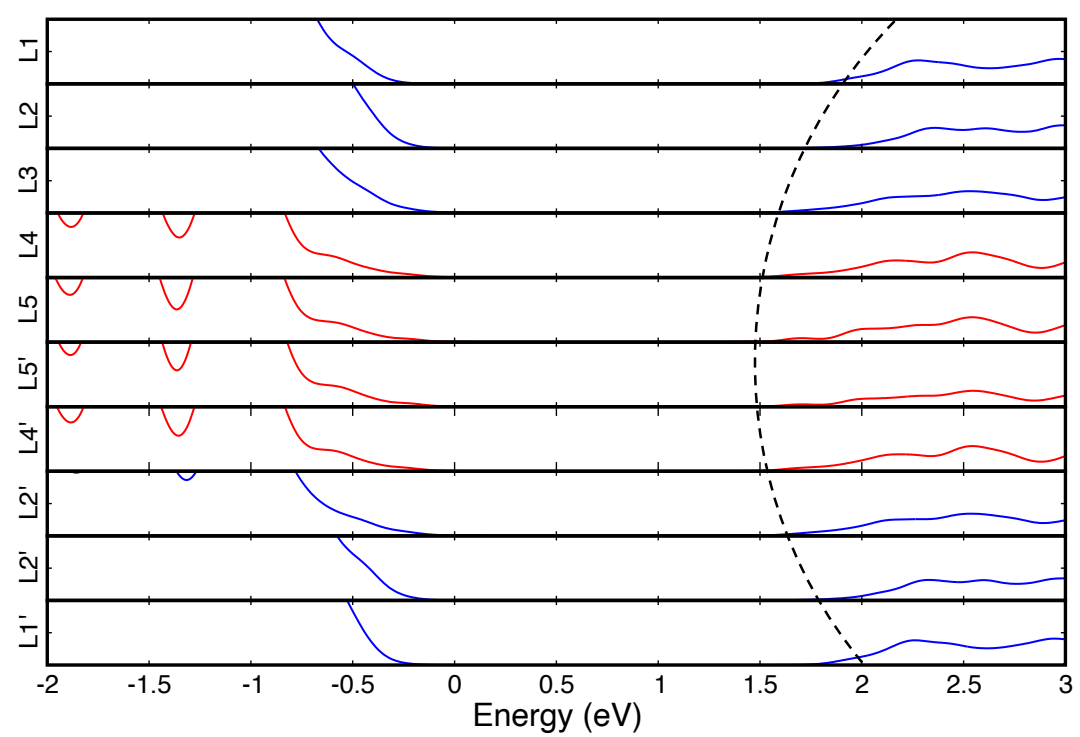

Figure $\mathbf{S 1 2}$ | a $\mathrm{CsPbl}_{3}$ slab with DMSO showing the naming of the layers for the projected density of states (PDOS). $\mathbf{b}$ Layer-by-layer PDOS of $\mathrm{CsPbl}_{3}$ slab with DMSO at the surfaces. PDOS of the inner bulk-like layers are shown in red. The dashed circular arc is a guide to the eye showing the widening of the band gap as one approaches the surface layers. The valence band maximum is set to zero. 
Figures $\mathbf{S 1 3}$ and $\mathbf{S} 14$ compare the band structures of $\mathrm{CsPb}_{3}$ slab with relaxed Csl-terminated surface and the slab terminated with a DMSO molecule, respectively. As one can see, the two band structures are quite similar and no surface states appear as a result of the introduction of DMSO (Figure S14) showing the passivating role of the latter.

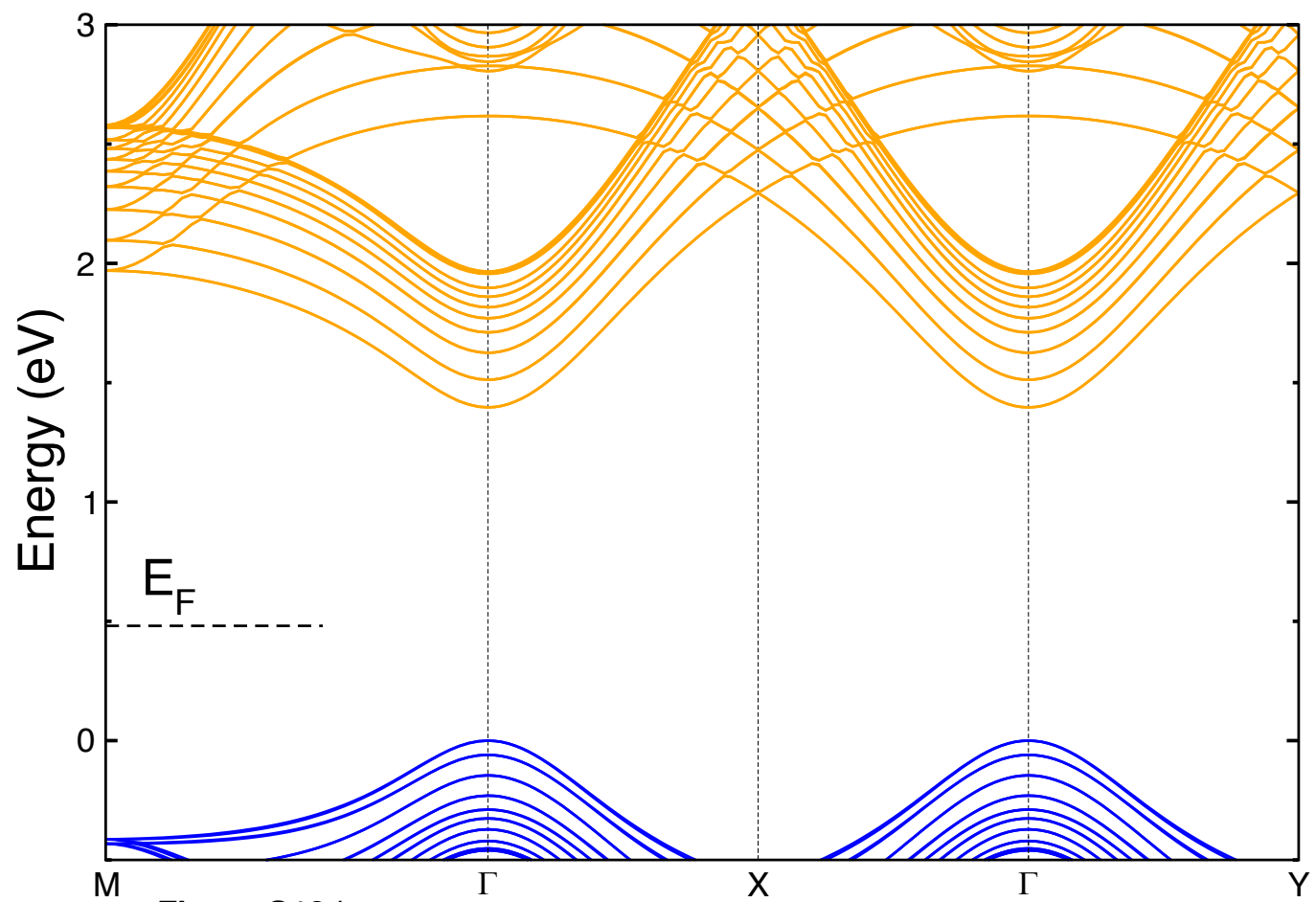

Figure S13 | Band structure of relaxed CsI-terminated $\mathrm{CsPb}_{3}$ slab surface.

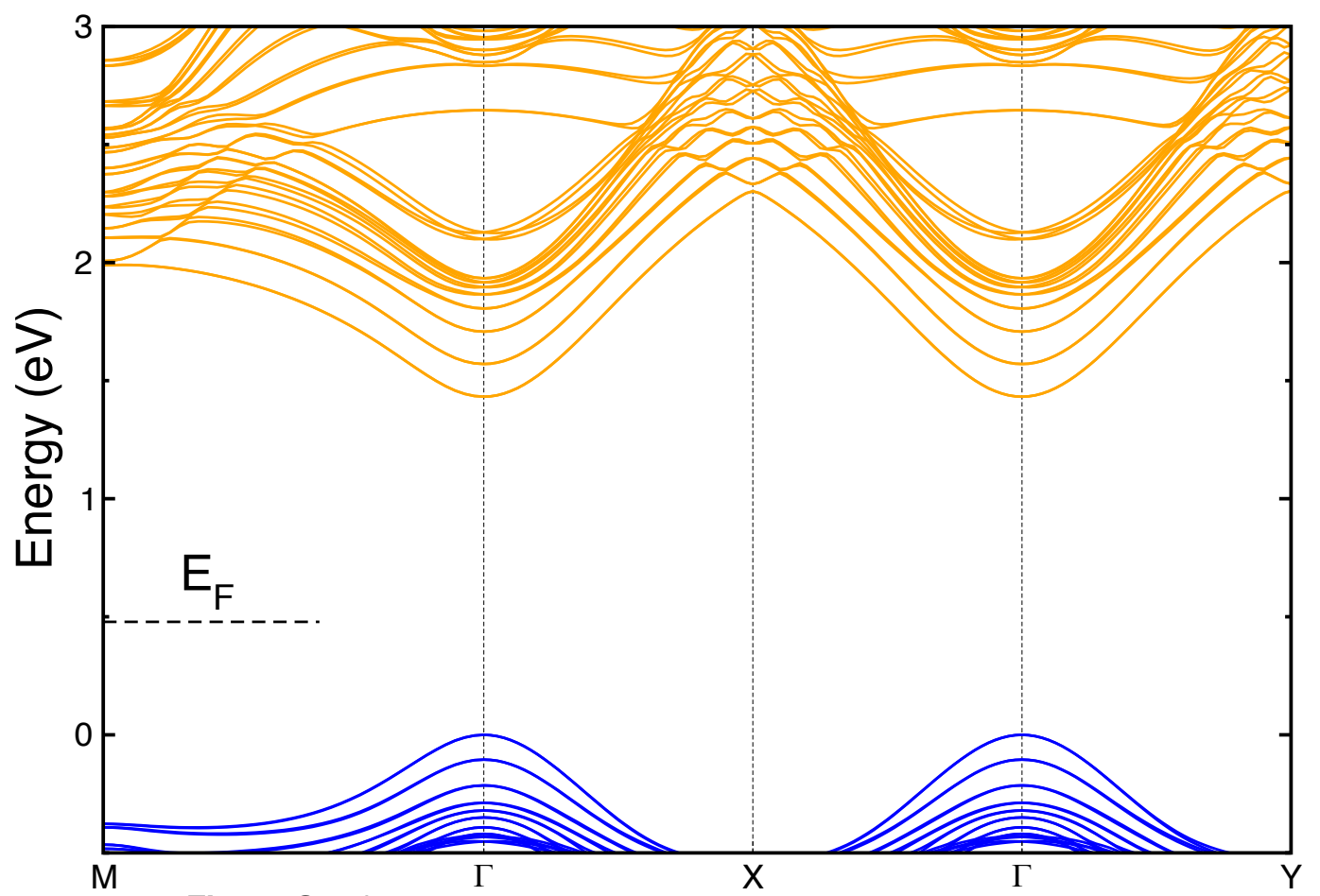

Figure $\mathrm{S} 14$ | Band structure of $\mathrm{CsPb}_{3}$ slab with DMSO at the surfaces. 


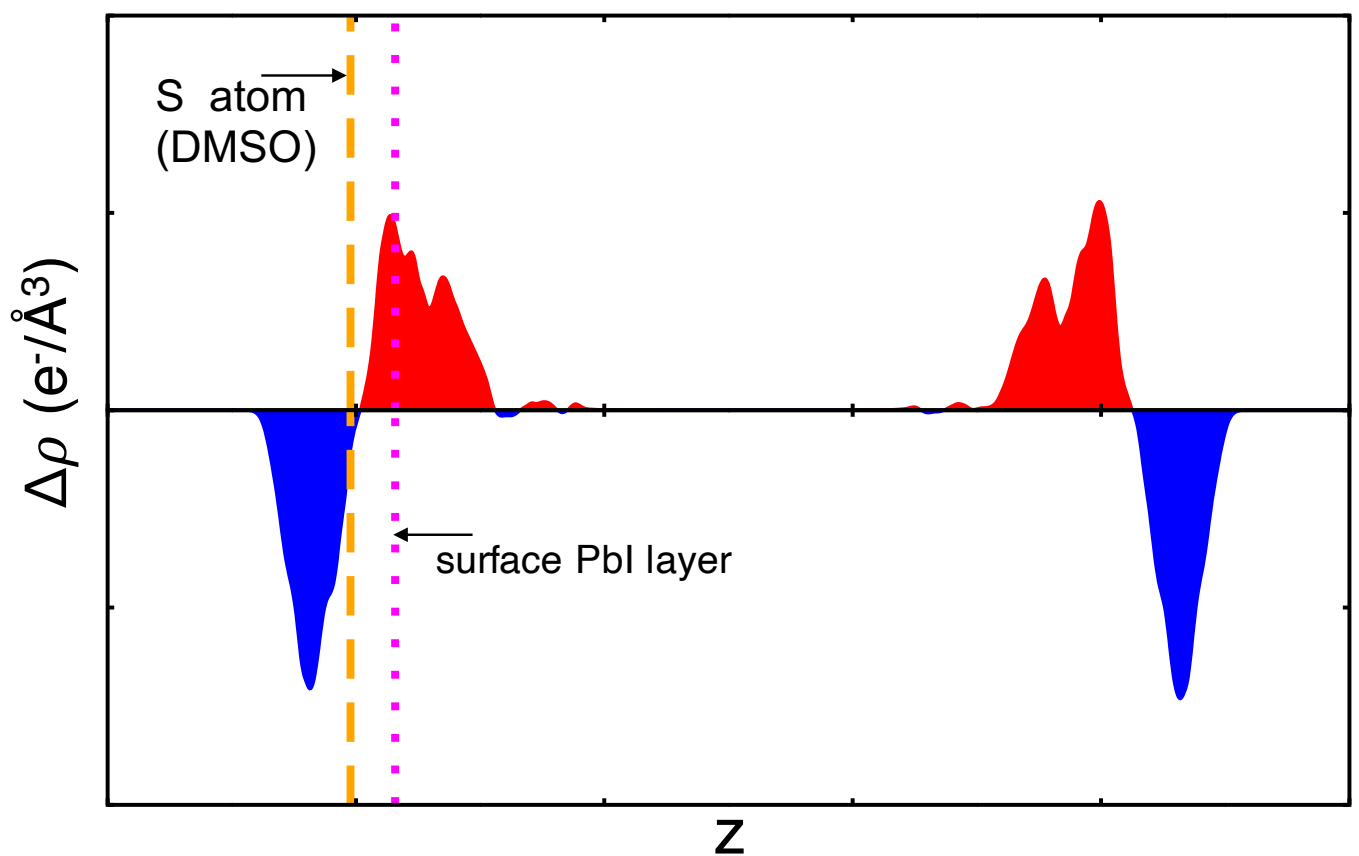

Figure S15 | Profile of total charge density transfered across the slab with DMSO at the CsI terminated surface of CsPbl 3 slab. Red for electronic charge accumulation and blue for electronic charge depletion. The positions of S atom from DMSO and surface Pbl layers are shown by vertical lines. Note that within SIESTA, the convention for total charge densities is: a positive sign is for electronic charge density and a negative sign is for ionic charge density.

\section{Surface dipoles with DMSO}

Considering the polarization profiles of Figure $3 \mathrm{c}$ of the manuscript and by integrating them, we obtain surface dipoles of about $2.52 \times 10^{-11} \mathrm{C} / \mathrm{m}(\sim 6.0 \mathrm{D})$ and $4.06 \times 10^{-11} \mathrm{C} / \mathrm{m}(\sim 9.7 \mathrm{D})$ for the slabs with DMSO and Csl termination, respectively. Hence, the resulting difference of $\sim 1.54 \times 10^{-11} \mathrm{C} / \mathrm{m}(\sim 3.7 \mathrm{D})$ represents the contribution of DMSO to the total surface dipole. In the isolated slabs with DMSO molecules on the different terminations, we obtain a surface dipole of $\sim 2.7-2.9 \mathrm{D}$ in fair agreement with the experimental value of $3.9 \mathrm{D}^{30}$. The calculated dipole moment for the isolated DMSO molecule is slightly lower than that obtained from the difference of the profiles of Figure $3 \mathrm{c}, \mathrm{e}$ of the main manuscript. We note that in the perovskite slab with DMSO, outer I and $\mathrm{Cs}\left(\mathrm{CsPb}_{3} \mathrm{slab}\right)$ or I and $\mathrm{MA}\left(\mathrm{MAPb}_{3} \mathrm{slab}\right)$ were removed, which partly explain this difference in addition to the contributions coming from the interactions of the molecule with the perovskite surface.

Remark: We note from a numerical point of view that the choice of the limits of integration for computing the surface dipoles (Eq 6 of the manuscript) somehow affects the individual computed surface dipole values because of the $z * \rho(z)$ product term. This tends to be more noticeable as the dipole becomes less vanishing in the bulk-like region of the slab and deep in the vacuum (due to more spurious charge densities in vacuum). The effect is less noticeable when similar slab sizes and limits of integration are used when comparing systems as we adopted in this work. However, the changes of surface dipoles $(\Delta p)$ are barely affected by these considerations and are the quantities of most interest in computing the changes in work functions $(\Delta \phi)$, which is the focus of our discussion. 


\section{Text S9 | Surface passivation with TMPPA}

Finally, we consider (2,4, 6-trimethylphenyl)phosphonic acid (TMPPA) to passivate the surfaces of both $\mathrm{CsPb}_{3}$ and $\mathrm{MAPbl}_{3}$ slabs. We use a similar approach as in DMSO to insert TMPPA at the surface. The relaxed structures with TMPPA at the surfaces are shown in Figure S16-S17 and the resulting band structures exhibit dispersive bands with no surface states inside the gap showing the passivating role of TMPPA (Figure S18-S19). In all cases, the presence of TMPPA at the surface pushes up the absolute valence energy levels of the different systems (Figure S20b-e) except for TMPPA at $\mathrm{Pbl}_{2}$-termination in $\mathrm{CsPbl}_{3}$ slab. The corresponding polarization density profiles clearly indicate a severe reduction of the surface dipole with the addition of TMPPA (Figure S20b,d,e and Table 1 of the manuscript). With TMPPA at $\mathrm{Pbl}_{2}$-termination in $\mathrm{CsPbl}_{3}$ slab, the change in surface dipole is less significant $\left(\sim 0.04 \times 10^{-11} \mathrm{C} / \mathrm{m}\right.$, Table 1 of the manuscript), which explains its similar $E_{v}^{a b s}$ as compared to $\mathrm{CsPbl}_{3}$ with $\mathrm{Pbl}_{2}$-termination. Notably, the differences $\Delta \mathrm{E}_{v}^{a b s}$ for all the different terminations are in excellent agreement with $\Delta \phi$ computed via Eq. 10 of the manuscript.

a

TMPPA on Csl surface

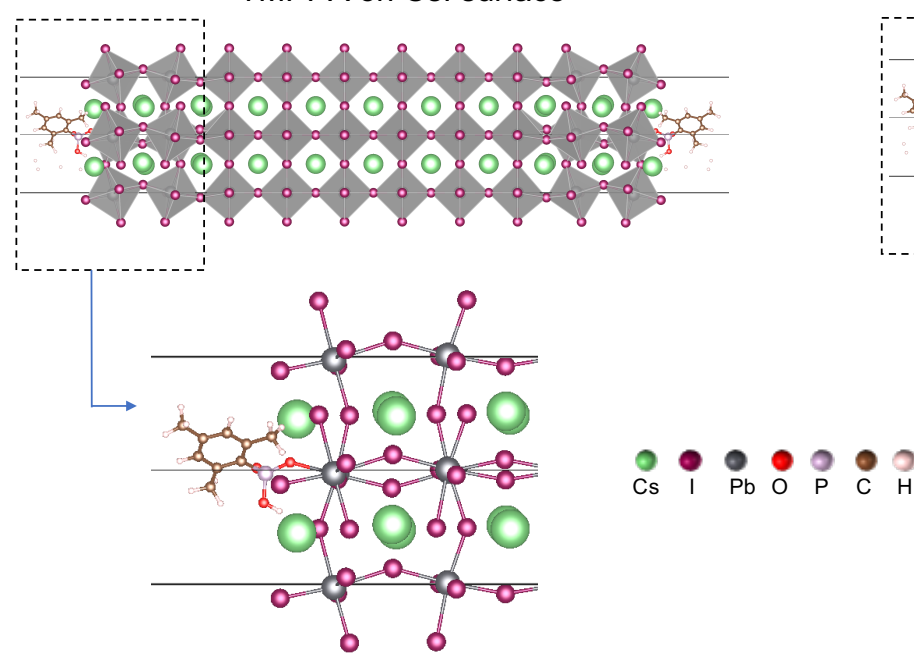

b

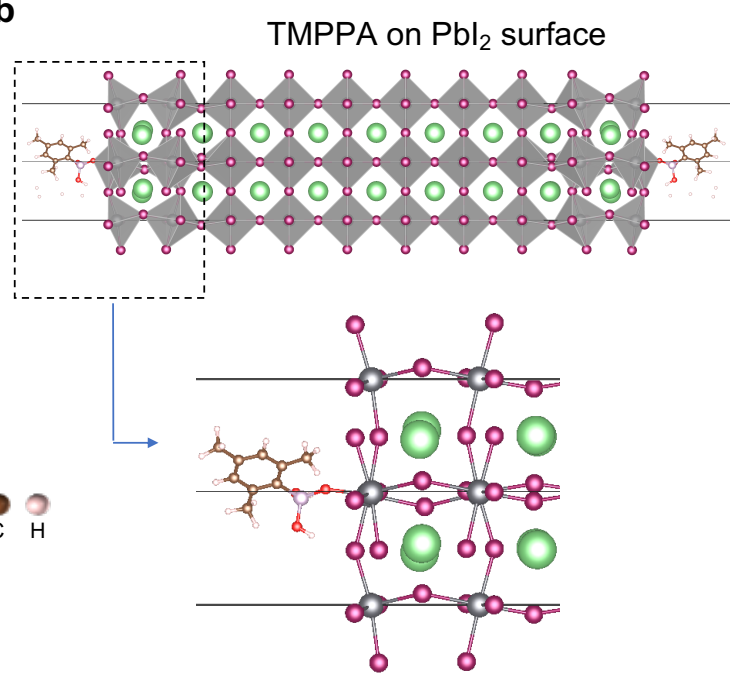

Figure S16 | Relaxed CsPbl 3 slabs with TMPPA on ' a the Csl-terminated surface $\mathbf{b}$ the $\mathrm{Pbl}_{2}$-terminated surface.

a

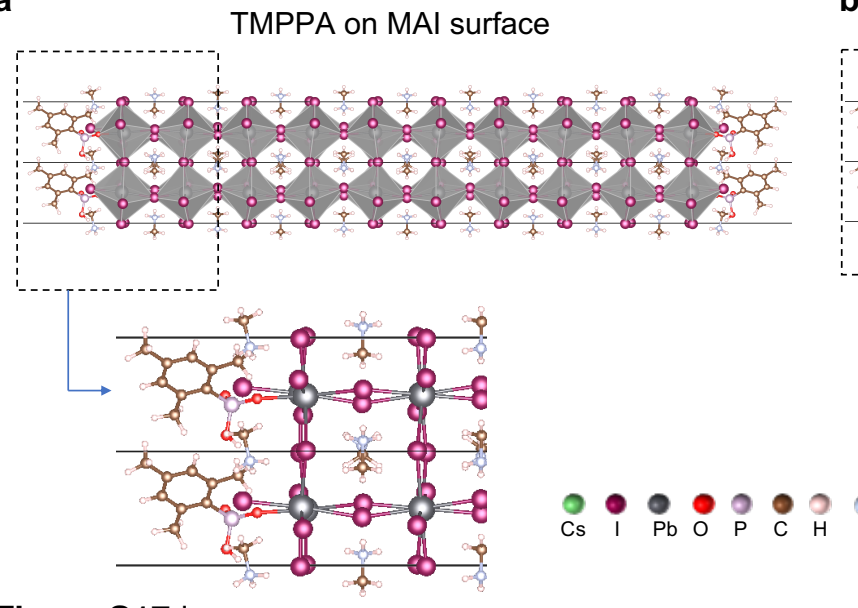

b

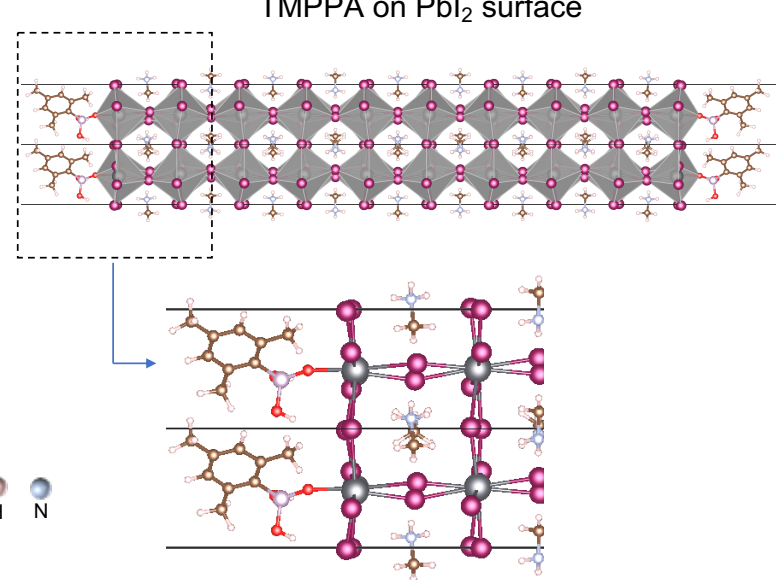

Figure $\mathbf{S 1 7}$ | Relaxed MAPbl 3 slabs with TMPPA on ' $\mathbf{a}$ the MAl-terminated surface $\mathbf{b}$ the $\mathrm{Pbl}_{2}$-terminated surface. 
Figures $\mathbf{S 1 8}$ and $\mathbf{S} 19$ compare the band structures of $\mathrm{CsPb}_{3}$ slab with relaxed Csl-terminated surface and the slab terminated with a TMPPA molecule, respectively. As one can see, the two band structures are quite similar and no surface states appear as a result of the introduction of TMPPA (Figure S14) showing the passivating role of the latter at this surface coverage.

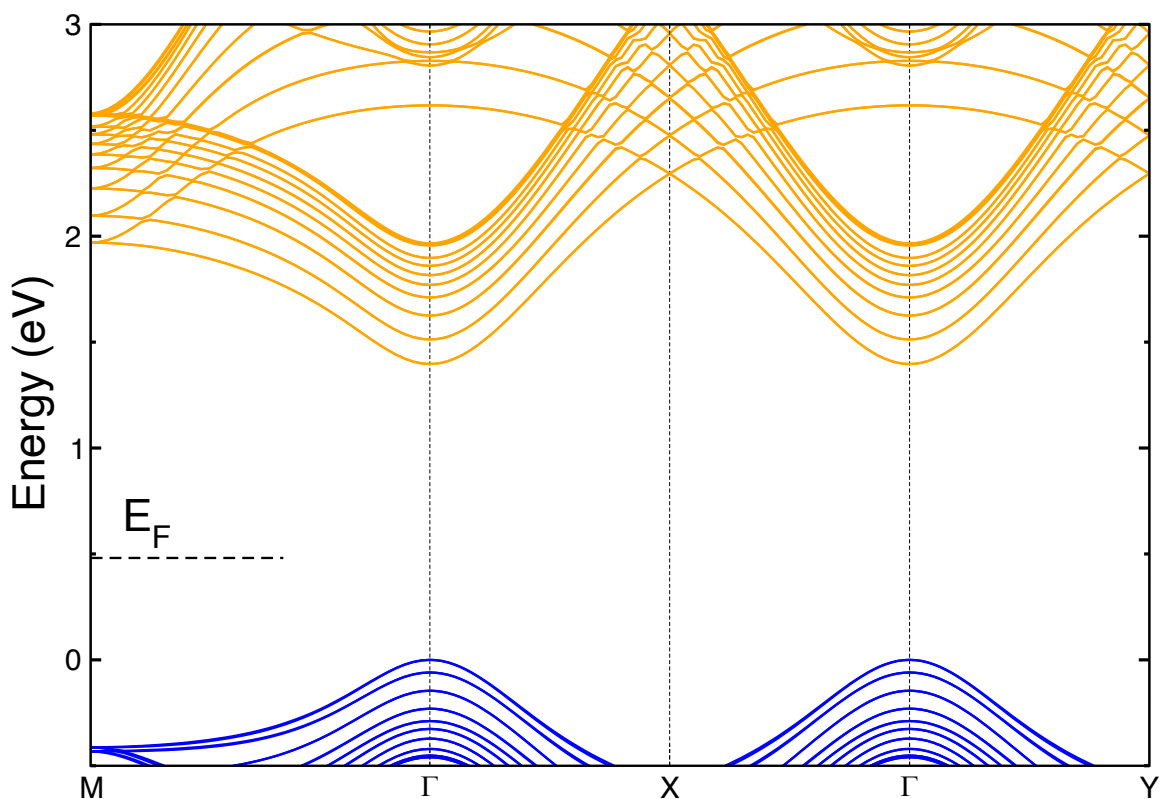

Figure S18 | Band structure of relaxed Csl-terminated $\mathrm{CsPbl}_{3}$ slab surface.

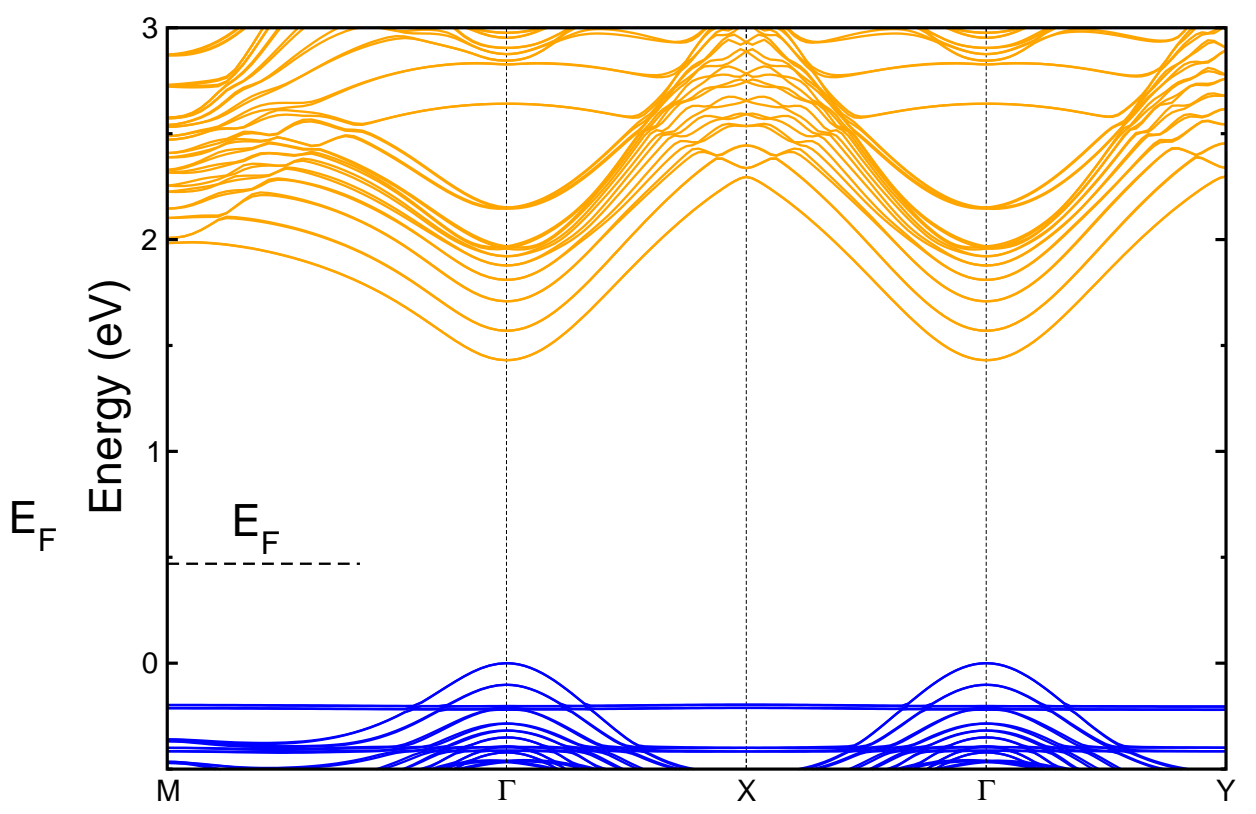

Figure S19 | Band structure of $\mathrm{CsPbl}_{3}$ slab with TMPPA at the surfaces. 


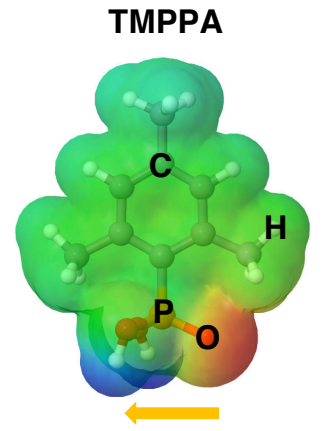

$\mathrm{CsPbl}_{3} \mathrm{slab}$

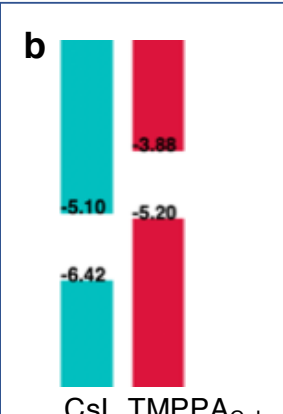

C

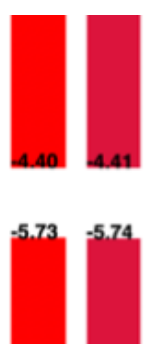

$\mathrm{Pbl}_{2}$ TMPPA $\mathrm{Pbl2}_{2}$
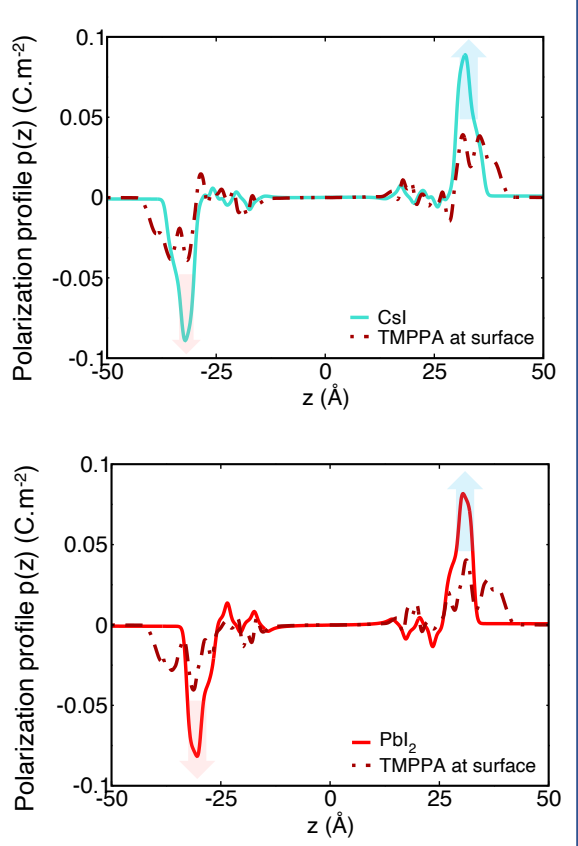

$\mathrm{MAPbl}_{3} \mathrm{slab}$

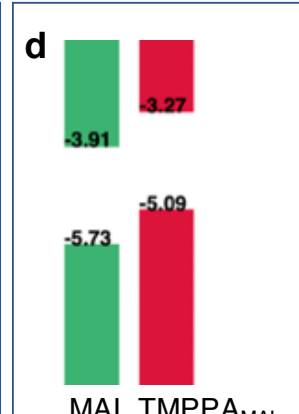

MAI TMPPA

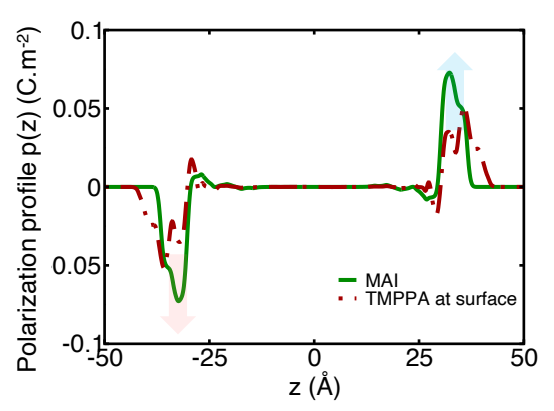

e

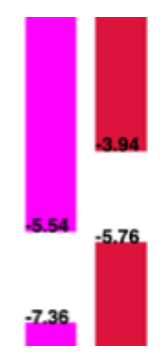

$\mathrm{Pbl}_{2}$ TMPPA

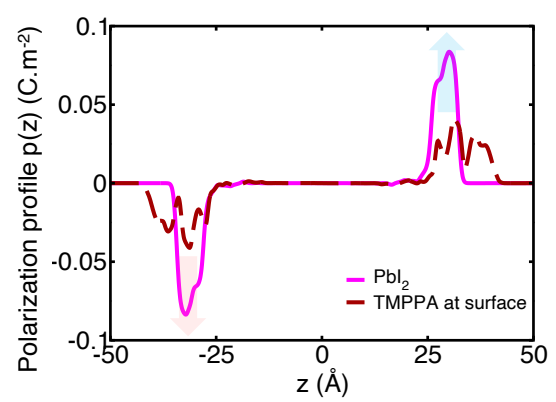

Figure S20 $\mid$ Effect of surface passivation and / or functionalization with TMPPA. a TMPPA molecule with the Hirshfeld net atomic charge map. Red for excess electronic charge and blue for electron deficiency. The arrow shows the direction of the dipole moment of TMPPA. b (left) Computed absolute valence energy levels of $\mathrm{CsPbl}_{3}$ slabs with CsI and TMPPA on CsI terminations. (right) Polarization density profiles comparing the surface dipoles of the slabs with CsI and TMPPA on CsI terminations. c (left) Computed absolute valence energy levels of $\mathrm{CsPbl}_{3}$ slabs with $\mathrm{Pbl}_{2}$ and TMPPA on Pbl $\mathrm{Tb}_{2}$ terminations. (right) Polarization density profiles comparing the surface dipoles of the slabs with $\mathrm{Pbl}_{2}$ and TMPPA on Pbl 2 terminations. d (left) Computed absolute valence energy levels of $\mathrm{MAPbl}_{3}$ slabs with MAI and TMPPA on MAI terminations. (right) Polarization density profiles comparing the surface dipoles of the slabs with MAI and TMPPA on MAI terminations. e (left) Computed absolute valence energy levels of $\mathrm{MAPbl}_{3}$ slabs with $\mathrm{Pbl}_{2}$ and TMPPA on $\mathrm{Pbl}_{2}$ terminations. (right) Polarization density profiles comparing the surface dipoles of the slabs with $\mathrm{Pbl}_{2}$ and TMPPA on $\mathrm{Pbl}_{2}$ terminations. 


\section{Text S10 | Additivity of the surface dipoles in (PEA) ${ }_{2} \mathrm{Pbl}_{4} / \mathrm{CsPbl}_{3}$ heterostructure}

Similar to the case of the heterostructure with BA molecule discussed in the main manuscript, one can see the additivity of the surface dipoles with $(\mathrm{PEA})_{2} \mathrm{Pbl}_{4} / \mathrm{CsPb}_{3}$ heterostructure as well. This indicates the generality of the approach.

a

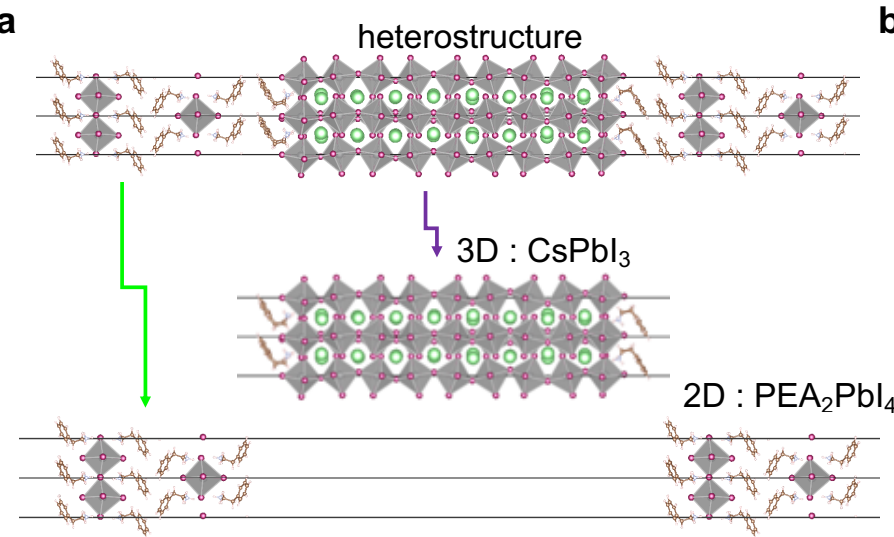

b

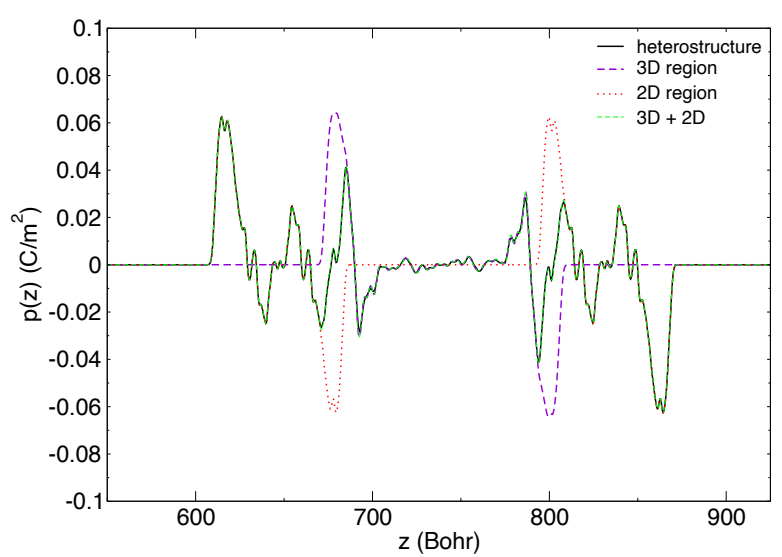

Figure S21 | Additivity of the surface dipoles. a The relaxed 2D/3D heterostructure model composed of $\mathrm{Cs}^{2} \mathrm{Pbl}_{3}$ interfaced with $(\mathrm{PEA})_{2} \mathrm{Pbl}_{3}$. The heterostructure is viewed as a composite showing its constituent parts. b Computed polarization density profiles of the $2 \mathrm{D} / 3 \mathrm{D}$ and the constituent parts demonstrating the additivity of the surface dipoles. 


\section{References}

[1] Hohenberg, P. \& Kohn, W. Inhomogeneous electron gas. Phys. Rev. 136, B864-B871 (1964).

[2] Kohn, W. \& Sham, L. J. Self-consistent equations including exchange and correlation effects. Phys. Rev. 140, A1133A1138 (1965).

[3] Soler, M. et al. The SIESTA method for ab initio order-N materials. J. Phys.: Condens. Matter 14, $2745-2779$ (2002).

[4] Artacho, E. et al. The SIESTA method; developments and applicability. J. Phys.: Condens. Matter 20, 064208 (2008).

[5] Cooper, V. R. Van der Waals density functional: An appropriate exchange functional. Phys. Rev. B 81, 161104 (2010).

[6] Hamada, I. \& Otani, M. Comparative van der waals density-functional study of graphene on metal surfaces. Phys. Rev. $B$ 82, 153412 (2010).

[7] Yuk, S. F. et al. Towards an accurate description of perovskite ferroelectrics: exchange and correlation effects. Sci. Rep. 7, 43482 (2017).

[8] Traore, B. et al. Importance of vacancies and doping in the hole-transporting nickel oxide interface with halide perovskites. ACS Appl. Mater. Interfaces 12, 6633-6640 (2020).

[9] Troullier, N. \& Martins, J. L. Efficient pseudopotentials for plane-wave calculations. Phys. Rev. B 43, 1993-2006 (1991).

[10] Perdew, J. P., Burke, K. \& Ernzerhof, M. Generalized gradient approximation made simple. Phys. Rev. Lett. 77, 38653868 (1996).

[11] Ferreira, L. G., Marques, M. \& Teles, L. K. Approximation to density functional theory for the calculation of band gaps of semiconductors. Phys. Rev. B 78, 125116 (2008).

[12] Fernández-Seivane, L., Oliveira, M. A., Sanvito, S. \& Ferrer, J. On-site approximation for spin-orbit coupling in linear combination of atomic orbitals density functional methods. J. Phys.: Condens. Matter 18, 7999-8013 (2006).

[13] Bitzek, E., Koskinen, P., Gähler, F., Moseler, M. \& Gumbsch, P. Structural relaxation made simple. Phys. Rev. Lett. 97, 170201 (2006). URL https://link.aps .org/doi/10.1103/PhysRevLett.97.170201.

[14] Marronnier, A. et al. Anharmonicity and disorder in the black phases of cesium lead iodide used for stable inorganic perovskite solar cells. ACS Nano 12, 3477-3486 (2018).

[15] Stoumpos, C. C. et al. Crystal growth of the perovskite semiconductor $\mathrm{cspbbr}_{3}$ : A new material for high-energy radiation detection. Cryst. Growth Des. 13, 2722-2727 (2013).

[16] Weller, M. T., Weber, O. J., Frost, J. M. \& Walsh, A. Cubic perovskite structure of black formamidinium lead iodide, $\alpha-\left[\mathrm{HC}\left(\mathrm{NH}_{2}\right)_{2}\right] \mathrm{Pbl}_{3}$, at 298 K. J. Phys. Chem. Lett. 6, 3209-3212 (2015).

[17] Billing, D. \& Lemmerer, A. Synthesis, characterization and phase transitions in the inorganic-organic layered perovskitetype hybrids $\left[\left(\mathrm{cnh}_{2 n+1} \mathrm{nh}_{3}\right)(2) \mathrm{pbi}_{4}\right], \mathrm{n}=4,5$ and 6. Acta Crystallogr., Sect. B: Struct. Sci. 63, 735-47 (2007).

[18] Fang, H.-H. et al. Band-edge exciton fine structure and exciton recombination dynamics in single crystals of layered hybrid perovskites. Adv. Funct. Mater. 30, 1907979 (2020).

[19] Bengtsson, L. Dipole correction for surface supercell calculations. Phys. Rev. B 59, 12301-12304 (1999). URL https: //link.aps.org/doi/10.1103/PhysRevB.59.12301.

[20] Meyer, B. \& Vanderbilt, D. Ab initio study of batio and pbtio $_{3}$ surfaces in external electric fields. Phys. Rev. B 63, 205426 (2001).

[21] Tao, S. X., Cao, X. \& Bobbert, P. A. Accurate and efficient band gap predictions of metal halide perovskites using the dft-1/2 method: Gw accuracy with dft expense. Sci. Rep. 7, 14386 (2017).

[22] Even, J., Pedesseau, L., Dupertuis, M.-A., Jancu, J.-M. \& Katan, C. Electronic model for self-assembled hybrid organic/perovskite semiconductors: Reverse band edge electronic states ordering and spin-orbit coupling. Phys. Rev. $B$ 86, 205301 (2012).

[23] Das, T., Rocquefelte, X. \& Jobic, S. Ab initio positioning of the valence and conduction bands of bulk photocatalysts: Proposition of absolute reference energy. J. Phys. Chem. C 124, 19426-19434 (2020). URL https://doi.org/10. 1021/acs.jpcc.0c04003. https://doi.org/10.1021/acs.jpcc.0c04003.

[24] Lodeiro, L. et al. Methodological issues in first-principle calculations of $\mathrm{ch}_{3} \mathrm{nh}_{3} \mathrm{pbi}_{3}$ perovskite surfaces: Quantum confinement and thermal motion. ACS Omega 5, 29477-29491 (2020).

[25] Knutson, J. L., , Martin, J. D. \& Mitzi, D. B. Tuning the band gap in hybrid tin iodide perovskite semiconductors using structural templating. Inorg. Chem. 44, 4699-4705 (2005). 
[26] Even, J., Pedesseau, L., Jancu, J.-M. \& Katan, C. Importance of spin-orbit coupling in hybrid organic/inorganic perovskites for photovoltaic applications. J. Phys. Chem. Lett. 4, 2999-3005 (2013).

[27] Traore, B. et al. Composite nature of layered hybrid perovskites: Assessment on quantum and dielectric confinements and band alignment. ACS Nano 12, 3321-3332 (2018).

[28] Katan, C., Mercier, N. \& Even, J. Quantum and dielectric confinement effects in lower-dimensional hybrid perovskite semiconductors. Chem. Rev. 119, 3140-3192 (2019).

[29] Petrov, A. A. et al. Formamidinium haloplumbate intermediates: The missing link in a chain of hybrid perovskites crystallization. Chem. Mater. 32, 7739-7745 (2020).

[30] Itoh, S. \& Ohtaki, H. A study of the liquid structure of dimethyl sulfoxide by the x-ray diffraction. Zeitschrift für Naturforschung A 42, 858-862 (1987). URL https://doi.org/10.1515/zna-1987-0816. 